\title{
WestVirginiaUniversity
}

THE RESEARCH REPOSITORY @ WVU

West Virginia Agricultural and Forestry Experiment

Davis College of Agriculture, Natural Resources

Station Bulletins

And Design

$1-1-1982$

\section{Meat pricing strategies and advertising : a case study}

Joyce Elaine Schumacher

Dale K. Colyer

Follow this and additional works at: https://researchrepository.wvu.edu/ wv_agricultural_and_forestry_experiment_station_bulletins

\section{Digital Commons Citation}

Schumacher, Joyce Elaine and Colyer, Dale K., "Meat pricing strategies and advertising : a case study" (1982). West Virginia Agricultural and Forestry Experiment Station Bulletins. 681T.

https://researchrepository.wvu.edu/wv_agricultural_and_forestry_experiment_station_bulletins/728 @ WVU. It has been accepted for inclusion in West Virginia Agricultural and Forestry Experiment Station Bulletins by an authorized administrator of The Research Repository@WVU. For more information, please contact ian.harmon@mail.wvu.edu. 
MEAT PRICING STRATEGIES AND ADVERTISAYG:

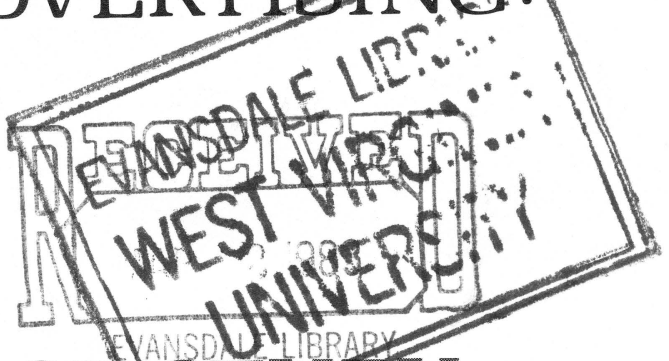

A CASEIST

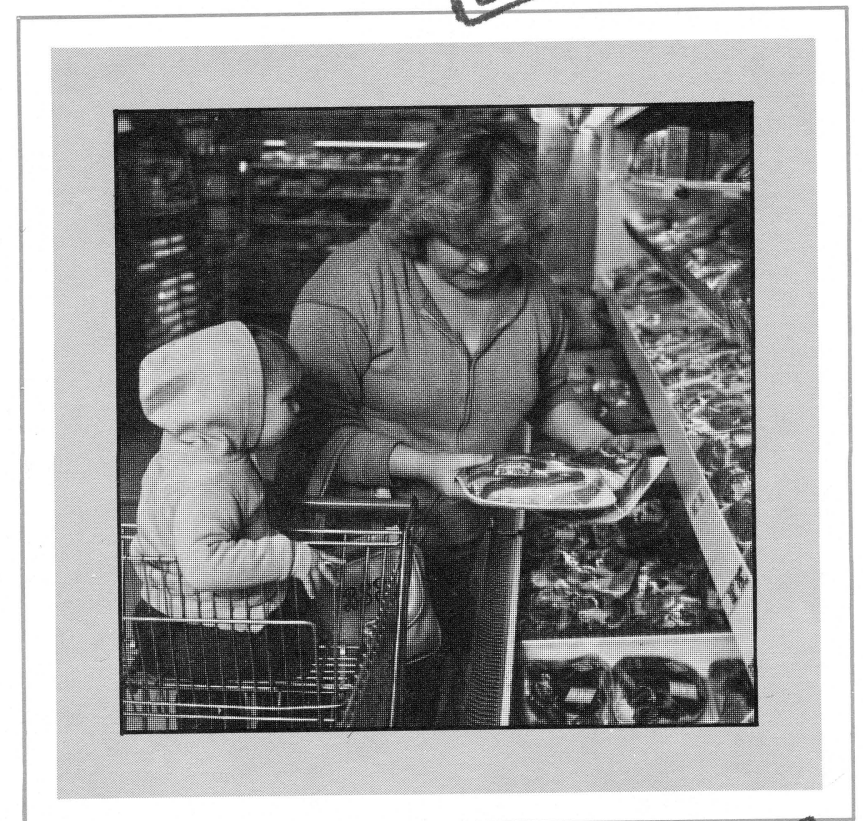

Bulletin 681T

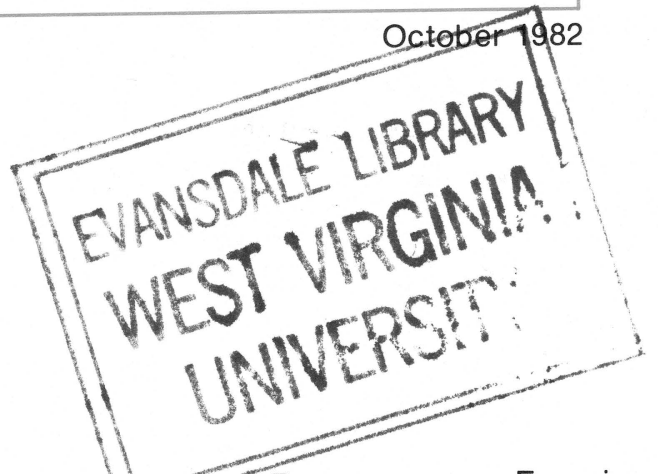

West Virginia University Agriculturaland Forestry Experiment Station 


\section{Authors}

Joyce E. Schumacher is a graduate research assistant in Agricultural Economics, and Dale Colyer is agricultural economist and chairman of the Division of Resource Management.

WEST VIRGINIA UNIVERSITY

AGRICULTURAL AND FORESTRY EXPERIMENT STATION COLLEGE OF AGRICULTURE AND FORESTRY

DALE W. ZINN, DIRECTOR

MORGANTOWN 


\section{Contents}

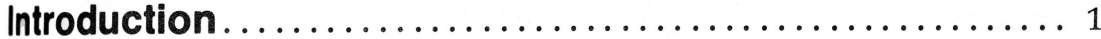

The Problem Statement ....................... 1

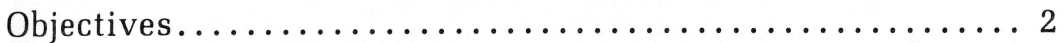

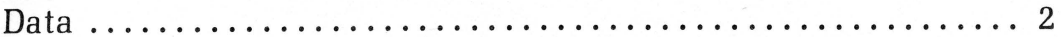

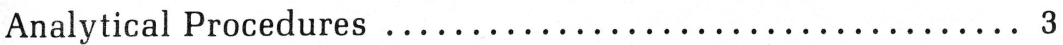

Meat Retail Pricing and Advertising Practices .......4

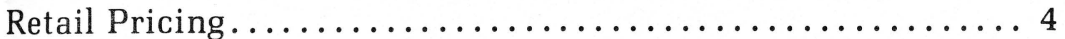

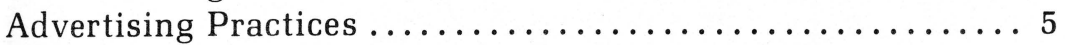

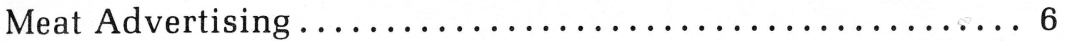

Pricing Study Results $\ldots \ldots \ldots \ldots \ldots \ldots \ldots \ldots \ldots \ldots \ldots$

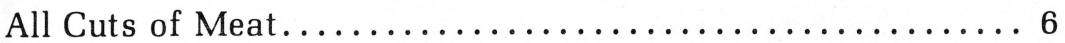

Selected Cuts of Meat......................... 8

Frequency of Advertising $\ldots \ldots \ldots \ldots \ldots \ldots \ldots \ldots \ldots \ldots$

Prices of Meats ............................... 11

Advertised Prices.......................... 11

Unadvertised Prices ........................ 17

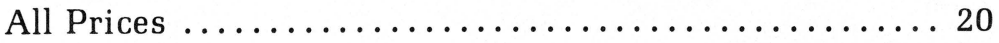

Type of Store......................... 26

Analysis of National Retail and Wholesale Prices .... 31

Retail Prices............................. 32

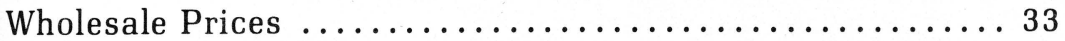

Lagged Price Models ......................... 33

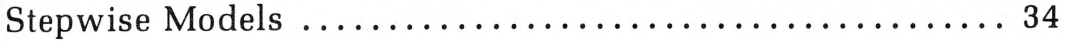

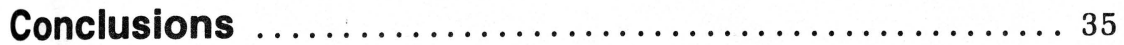

Bibliography $\ldots \ldots \ldots \ldots \ldots \ldots \ldots \ldots \ldots \ldots \ldots \ldots \ldots \ldots \ldots \ldots$

Appendix .................................. 39 
Blank Page in Original Bulletin 


\section{Meat Pricing Strategies and Advertising: A Case Study}

\section{Joyce E. Schumacher and Dale Colyer}

\section{Introduction}

Food retailing is an important component of the American economy. It is a complex industry in which the farmer, the processor and distributor are integrally related. Consumers spent $\$ 262$ billion on food grown on U.S. farms in 1980. The marketing bill, the difference between the amount the final consumers pay for a product and the amount producers of the product receive, accounted for $\$ 182$ billion of this total expenditure. Within the marketing bill, advertising accounted for 2 percent of the total, or $\$ 3.64$ billion (USDA, 1980).

Food retailing is a big business and will continue to grow in the 1980 s as it did throughout the 1970s. The pricing behavior of the numerous food retailers is highly sophisticated as each retailer bids for the consumer's dollars. Pricing strategies and merchandising policies become more complex as food retailing departs from a purely competitive environment.

\section{The Problem Statement}

Within the grocery retailing industry, advertising is used as a tool to increase profits, increase sales, and to differentiate products. It also is common to advertise other services such as carry-out service, friendly clerks, complete "deli," check cashing, and extended hours. However, consumers may be misled and/or confused by advertising. Each week the consumer is bombarded by advertising through the mass media, including newspapers. Some of this advertising provides objective information about the product, but many advertisements are meant to be persuasive, appealing to the subconscious or desires and predilections of the consumer. Furthermore, it may be difficult for a consumer to determine if the advertised prices are lower, higher, or the same as regular prices in the supermarket. Today's consumers need be aware of and understand advertising practices in the local market so that they can maximize the use of their purchasing power. 
The local newspaper is a common exposure that consumers in a local market area have to grocery advertising. Advertised products include man y items in the consumer market basket of which meat is an important component. Meat, poultry, and eggs account for 32.2 percent of all food in the consumer price index (Manchester). Hence, the grocery retailer can use the advertising of meat prices to attract a consumer to the store.

\section{Objectives}

This study was undertaken to provide insights into the relationships that exist between meat pricing and advertising strategies. The general objective was to obtain information about meat advertising practices. The specific objectives were to:

1. Determine how often different cuts of meat are advertised;

2. Determine if there are significant differences between advertised prices of meats as opposed to the regular prices;

3. Determine if there are significant differences between the average meat prices of grocery stores in the same local market area;

4. Determine if significant differences between the average meat prices of chains, voluntary chains and independent stores exist;

5. Determine if there are relationships between national retail/wholesale meat prices and local retail meat prices.

\section{Data}

The primary data used for this study were collected in Morgantown, West Virginia, from grocery stores and newspapers for a period of 34 consecutive weeks in 1978 (March-October). The city of Morgantown is located in the Pittsburgh Market Area as defined by the Progressive Grocer's Marketing Guidebook. The 1980 Preliminary U.S. Census of Population indicated that the Morgantown area (including the towns of Star City and Westover) had a population of 33,902 (U.S. Department of Commerce).

Using the definitions of Progressive Grocer, the Morgantown market area consists of nine supermarkets, of which five are chain stores, three are voluntary chain stores, and one is an independent store. A chain store is one in which eleven or more retail stores are under one ownership, whereas an independent store operates in an organization with less than eleven stores. Voluntary chains are "retailers who belong to voluntary merchandising groups sponsored by wholesalers and who operate under a common name." All nine 
supermarkets in the Morgantown market area were included in this study. ${ }^{1}$

One local daily paper (The Dominion Post) serves the area. Its daily circulation is approximately 21,000 , with a Sunday circulation of approximately 25,000 . During this study, all daily advertised prices of different cuts of fresh beef, chicken, and pork were enumerated from the advertisements in the local daily paper and the Sunday edition.

Each week, each of the nine stores was visited and prices of the different cuts of meat were recorded from meat in the display cases. (It was assumed that all stores carried beef that was USDA Grade Choice, chicken that was USDA Grade A and pork that was U.S. No. 1.) Data on a large number of cuts of meat were recorded, but for purposes of comparison only the meat cuts that could be found in common in most of the stores were used for the complete analysis.

Data were obtained from the USDA on the weekly wholesale and retail prices of beef and pork. Chicken prices are not summarized on a weekly basis. The wholesale price is the value (price) of the wholesale quantity equivalent of one pound of retail cuts. The retail value (price) is the estimated weighted average price of retail cuts from the carcass (USDA, February, 1980).

\section{Analytical Procedures}

Descriptive statistics were employed to characterize the data set. To determine the manner in which the price measurements were distributed, frequency counts were performed. When necessary because of a varying number of observations, the frequency counts were converted to percentages. Averages were calculated to help describe the frequency distribution and provide a measure of central tendency.

A Duncan's Multiple Range Test (DMRT) was used to determine if there were significant differences between the mean prices of the various cuts of meat. The DMRT “... takes into account the number of treatments in the experiment ... it permits decision as to which differences are significant and which are not . . . it uses a set of significant ranges, each range depending upon the number of means in the comparison" (Steel and Torries, p. 109).

Regression models were utilized to determine if linear relationships exist between the local retail prices of the selected cuts of meat and the

'In this study, two stores, Stores 2 and 3, are in the same voluntary chain. Two stores, Stores 6 and 7, represent the same regional chain, and two stores, Stores 4 and 5, are in the same national chain. 
USDA's estimated weighted average price of meat. Model I was used to estimate the relationships between the local retail prices of the selected cuts of meat and the USDA's estimated weighted average price of retail beef and pork cuts from the carcass. Model II was used to estimate the relationships between the local retail price of the selected meat cuts and the USDA's value of wholesale quantity equivalent to one pound of retail cuts. Model III was developed to discover if a linear relationship existed between the local retail prices and the national weighted average price of selected retail beef cuts lagged one week. Model IV was similar to Model III but the local retail prices were compared to the wholesale prices.

Models V and VI used a stepwise regression procedure where lagged variables for up to five weeks were added to Models I and II. In the stepwise procedure, variables are added one by one provided the variable is found to be statistically significant at a predetermined level of probability. These models were used to determine if local retail prices lagged behind either national retail or wholesale prices.

\section{Meat Retail Pricing and Advertising Practices}

This section contains a brief review of the available literature that pertains specifically to the advertising of retail meat prices, a general discussion on retail pricing behavior, a discussion of advertising practices, and a review of the current literature available on the advertising of retail meat prices.

\section{Retail Pricing}

Pricing is a complex arena where there are no exact decision tools and thus it is subject to executive judgments. The selected pricing policy can help make or break user responses to a product. Thus, pricing should be one of the more important decisions made by a firm's manager.

Pricing procedures and policies of a particular firm will vary according to the firm's marketing and company objectives. They can be used to increase sales of products. This is associated with volume objectives. Some firms seek to reach a maximum level of sales while maintaining a predetermined rate of return on investment. Another volume objective is to increase a firm's share of the market. By manipulating prices and increasing volume, a firm can obtain a more favorable position in the marketplace and help ensure its survival in the long run. 
In addition to volume objectives, a firm usually has profit objectives. One common objective is to obtain a predetermined rate of return. Many factors enter into determining this rate of return and the rate is often based on the type of industry, type of product, fiscal policy, financial structure, and the degree of risk involved (Kelley). In classical economics, the pricing objective is to maximize profit. Here economists have shown profit maximization exists where marginal revenue equals marginal cost. However, most businessmen usually use a cost-plus approach. This approach determines a per unit cost and then adds a markup that is intended to cover fixed and additional variable costs plus a profit. In the grocery retailing industry, the markup or margin on product lines varies (Rhodes).

Historically, higher margins in meat and produce departments have been used by grocery retailers to offset low margins in other departments or for items on sale. Because meats tend to have large margins and are an important component of the market basket, meat prices are often reduced in the expectation of improving the total profit of a particular store.

\section{Advertising Practices}

The formulation of pricing strategies and procedures is complex and interdependent with other elements in the marketing mix, including advertising strategies. Advertising is important because it can attract attention and interest, inform or persuade, and finally lead to selling the product. For a successful campaign, the advertiser usually analyzes the market for demographic characteristics such as income, age, sex, and education. From this information the appropriate advertising strategies and media are chosen.

In a local market area, newspapers frequently are chosen as an effective way to reach a broad and specific audience. Newspapers accounted for approximately 20 percent of the advertising dollars spent during 1979 (Gallo, 1981). Advantages of this approach are relatively low costs and the serving of a target population. Food retailers use newspaper advertisements to make consumers aware of the prices on various items. It is common to see a "loss leader" used in a competitive market area. A loss leader is a very low price, which may be at or below cost, and which is used to attract customers to a store with the expectation that they will buy items other than what is advertised. The store assumes that the regular prices on the other items will make up for the losses incurred on the loss leader. 


\section{Meat Advertising}

Gray and Anderson, for a case study in Palo Alto, California, during 1960, examined advertised prices recorded from weekly newspapers on many items found in the market basket. In 92 percent of their observations the advertised price of an item was lower in the stores that advertised than in those that did not. Furthermore, the average of the advertised price was found to be approximately 20 percent less.

Rhodes and Abou-Bakr found, from a study conducted on meat items in Columbia, Missouri, that the mean of advertised prices averaged 91.2 percent of non-advertised prices. The pricing policies of chain stores involved in their study suggested that the stores tried to attract customers by maintaining everyday low prices and by advertising the same competitive price for several weeks. Hence, the differences between their advertised and non-advertised prices were relatively small. The affiliated stores in their study usually had greater reductions for their advertised prices, but higher regular prices.

Rhodes, et al., in Customer Responses to Retail Meat Prices and Ads found two merchandising strategies for meat items. One store had an everyday low price strategy and advertised competitive prices. The second practiced advertising meats as weekly specials with greater price reductions, but had higher regular meat prices. Furthermore, it was found that advertised price reductions did result in a greater response. The store in which 58 percent of its advertised specials were price reductions had a larger sales response than the store where only 13 percent of its advertised specials were actual price cuts.

\section{Pricing Study Results}

The primary data were tabulated and frequency counts made for all cuts of meat for which prices were collected. Then, data on selected cuts of meat were tabulated and analyzed. An analysis and interpretation of prices of the selected cuts of meat grouped by advertised, unadvertised, and combined meat prices was completed. Finally, an analysis of the prices was made by type of store.

\section{All Cuts of Meat}

Frequency tables were developed from the primary data for each of the nine supermarkets and for all meats. The frequency counts for advertised meat items, unadvertised meat items, and the combined frequencies are shown in Table 1. 
Table 1.

Frequency of Advertising for All Meats by Type of Meat and Store

\begin{tabular}{|c|c|c|c|c|c|c|c|c|c|}
\hline & $\begin{array}{c}\text { Regional } \\
\text { Chain } \\
(1) \\
\end{array}$ & $\begin{array}{c}\text { Voluntary } \\
\text { Chain } \\
(2) \\
\end{array}$ & $\begin{array}{c}\text { Voluntary } \\
\text { Chain } \\
(3)\end{array}$ & $\begin{array}{c}\text { National } \\
\text { Chain } \\
(4)\end{array}$ & $\begin{array}{c}\text { National } \\
\text { Chain } \\
(5)\end{array}$ & $\begin{array}{c}\text { Regional } \\
\text { Chain } \\
(6)\end{array}$ & $\begin{array}{c}\text { Regional } \\
\text { Chain } \\
(7) \\
\end{array}$ & $\begin{array}{c}\text { Independent } \\
(8)\end{array}$ & $\begin{array}{c}\text { Voluntary } \\
\text { Chain } \\
(9) \\
\end{array}$ \\
\hline \multicolumn{10}{|l|}{ ALL MEATS } \\
\hline Advertised & 319 & 165 & 162 & 269 & 259 & 218 & 215 & 303 & 396 \\
\hline Unadvertised & 1199 & 1135 & 1265 & 1000 & 1144 & 959 & 945 & 561 & 950 \\
\hline Total & 1518 & 1300 & 1427 & 1269 & 1403 & 1177 & 1160 & 864 & 1346 \\
\hline Percent & 21 & 13 & 11 & 21 & 18 & 19 & 19 & 35 & 29 \\
\hline \multicolumn{10}{|l|}{ PORK } \\
\hline Advertised & 106 & 31 & 31 & 53 & 49 & 31 & 32 & 64 & 90 \\
\hline Unadvertised & 253 & 167 & 237 & 185 & 305 & 180 & 157 & 79 & 116 \\
\hline Total & 359 & 198 & 268 & 238 & 354 & 211 & 189 & 143 & 206 \\
\hline Percent & 30 & 16 & 12 & 22 & 14 & 15 & 17 & 45 & 44 \\
\hline \multicolumn{10}{|l|}{ CHICKEN } \\
\hline Advertised & 29 & 44 & 43 & 69 & 69 & 11 & 11 & 56 & 106 \\
\hline Unadvertised & 262 & 286 & 263 & 198 & 228 & 263 & 236 & 128 & 150 \\
\hline Total & 291 & 330 & 306 & 267 & 297 & 274 & 247 & 184 & 256 \\
\hline Percent & 10 & 13 & 14 & 26 & 23 & 4 & 4 & 30 & 41 \\
\hline \multicolumn{10}{|l|}{ BEEF } \\
\hline Advertised & 184 & 90 & 88 & 147 & 141 & 176 & 172 & 183 & 200 \\
\hline Unadvertised & 684 & 682 & 765 & 617 & 611 & 516 & 552 & 354 & 684 \\
\hline Total & 868 & 772 & 853 & 764 & 752 & 692 & 724 & 537 & 884 \\
\hline Percent & 21 & 12 & 10 & 19 & 19 & 25 & 24 & 34 & 23 \\
\hline
\end{tabular}


Due to the varying number of observations, the frequency counts were converted to percentages to allow meaningful comparisons. The equation used was:

Eq. $1 \frac{\text { Advertised Frequency }}{\begin{array}{c}\text { Advertised } \\ \text { Frequency }\end{array}+\begin{array}{c}\text { Unadvertised } \\ \text { Frequency }\end{array}} \times 100=$ Percent Advertised

The table shows that when all meats are considered, one regional chain, the independent, and one of the voluntary chains advertised more often than the other stores. Each of those three stores advertised more than 300 cuts during the 34 -week period. The other voluntary chain (which had two stores in the study) advertised much less than the other stores with an average of only 163 times compared to an overall average of 256 times. The differences in the frequency of total cuts advertised in each store ranged from 162 times to 396 times. Since these frequencies included all fresh meat items, the same information was tabulated by store for the meat items grouped by pork, chicken, and beef. The same independent and voluntary chain are among the stores that frequently advertise each meat type. The other voluntary chain is among the stores that advertise each meat type less frequently. Furthermore, it was found that beef is much more heavily advertised, both in number of cuts and frequency per cut, than either pork or chicken. Advertised prices for beef accounted for 60 percent of all advertised prices while chicken advertisements were 19 percent of the total and pork advertisements were 21 percent of the total.

\section{Selected Cuts of Meat}

The primary data consisted of numerous cuts of meat for which the availability and frequency of advertising varied considerably among the stores. Therefore, only those meat cuts that were found to be relatively common in most of the stores were used for the rest of this analysis. These included six cuts of pork, seven cuts of chicken, and ten cuts of beef. The selected cuts are shown in Table 2 .

\section{Frequency of Advertising}

Data from all nine stores were combined and counts for advertised meats and unadvertised meats were developed. Again, due to a varying number of observations, the frequencies were converted to percentages. Based on this data, the consumer can expect to find spare ribs, pork steak, ground beef, stew meat, and round steak to be advertised in the local area about one in four weeks. Cuts such as 
Table 2.

Frequency of Advertised and Non-Advertised Prices of Selected Cuts of Meat

Non-

Percent

Meat Item Advertised Advertised Advertised

PORK

Loin end roast

19

80

19

Rib center cut pork chops

12

176

6

Picnic

25

38

40

Loin center cut pork chops

Spare ribs

7

219

41

122

3

Pork steak

22

85

25

CHICKEN

Drumsticks

25

235

10

Fryer

47

228

17

Mixed parts

106

75

59

Roasting chicken

Thighs

24

144

14

34

237

13

11

147

Whole cut-up

22

219

9

BEEF

Ground beef

70

229

23

Ground chuck

56

264

18

Ground round

Porterhouse steak

13

198

39

221

6

Sirloin steak

27

183

15

Stew

71

190

13

T-Bone steak

38

214

27

Rump roast

Chuck roast

59

135

15

10

75

30

61

184

12

Round steak

rump roasts and pork picnics were advertised more frequently than most other meats. Of all the meat cuts studied, however, mixed fryer parts was the most frequently advertised meat item in the local area, with advertisements appearing 59 percent of the time.

Frequency tables for each of the nine supermarkets for all meats selected for this study are reported in Table 3 . The table shows that the two national, the independent, and one of the voluntary chains 
Table 3.

Frequency of Advertising for Selected Meats by Type of Meat and Store

\begin{tabular}{|c|c|c|c|c|c|c|c|c|c|}
\hline & $\begin{array}{c}\text { Regional } \\
\text { Chain } \\
(1)\end{array}$ & $\begin{array}{c}\text { Voluntary } \\
\text { Chain } \\
(2)\end{array}$ & $\begin{array}{c}\text { Voluntary } \\
\text { Chain } \\
(3)\end{array}$ & $\begin{array}{c}\text { National } \\
\text { Chain } \\
(4)\end{array}$ & $\begin{array}{c}\text { National } \\
\text { Chain } \\
(5)\end{array}$ & $\begin{array}{c}\text { Regional } \\
\text { Chain } \\
(6) \\
\end{array}$ & $\begin{array}{c}\text { Regional } \\
\text { Chain } \\
(7) \\
\end{array}$ & $\begin{array}{c}\text { Independent } \\
(8)\end{array}$ & $\begin{array}{c}\text { Voluntary } \\
\text { Chain } \\
(9)\end{array}$ \\
\hline \multicolumn{10}{|l|}{ ALL MEATS } \\
\hline Advertised & 61 & 79 & 77 & 127 & 125 & 66 & 62 & 102 & 167 \\
\hline Unadvertised & 430 & 438 & 535 & 393 & 369 & 538 & 531 & 248 & 373 \\
\hline Total & 491 & 517 & 612 & 520 & 494 & 604 & 593 & 350 & 540 \\
\hline Percent & 12 & 15 & 13 & 24 & 25 & 11 & 10 & 29 & 31 \\
\hline \multicolumn{10}{|l|}{ PORK } \\
\hline Advertised & 6 & 9 & 9 & 20 & 19 & 13 & 12 & 9 & 29 \\
\hline Unadvertised & 68 & 40 & 97 & 99 & 78 & 124 & 120 & 34 & 46 \\
\hline Total & 74 & 49 & 106 & 119 & 97 & 137 & 132 & 43 & 75 \\
\hline Percent & 8 & 18 & 8 & 17 & 20 & 9 & 9 & 21 & 39 \\
\hline \multicolumn{10}{|l|}{ CHICKEN } \\
\hline Advertised & 7 & 32 & 31 & 63 & 63 & 10 & 10 & 21 & 59 \\
\hline Unadvertised & 157 & 181 & 173 & 124 & 126 & 171 & 166 & 74 & 113 \\
\hline Total & 163 & 213 & 204 & 187 & 189 & 181 & 176 & 95 & 172 \\
\hline Percent & 4 & 15 & 15 & 34 & 33 & 6 & 6 & 22 & 34 \\
\hline \multicolumn{10}{|l|}{ BEEF } \\
\hline Advertised & 48 & 38 & 37 & 44 & 43 & 43 & 40 & 72 & 79 \\
\hline Unadvertised & 205 & 217 & 265 & 170 & 165 & 243 & 245 & 140 & 214 \\
\hline Total & 253 & 217 & 302 & 114 & 208 & 286 & 285 & 212 & 293 \\
\hline Percent & 19 & 15 & 12 & 39 & 21 & 15 & 14 & 34 & 27 \\
\hline
\end{tabular}


advertised more frequently than the other stores when all three types of meat are considered. Each of those three stores advertised more than 100 cuts during the 34 -week period. When meat items are grouped by type (pork, chicken, and beef) the same stores which advertised meats more frequently tended to advertise chicken and beef more than the other stores, along with one of the voluntary chains which advertised pork relatively more frequently. Pork cuts, in general, tended to be advertised less frequently, only 15 percent, as compared to chicken, 34 percent, and to beef, 51 percent. Some of the differences are due to the larger number of beef cuts finally selected for this study. However, as indicated previously, when all cuts of each meat were used, it was found that beef was advertised much more frequently than pork or chicken.

\section{Prices of Meats}

Tables 4 and 5 show average prices in cents per pound of all the selected meat items, with the meat items as rows and the stores as columns. Table 4 contains the means of the unadvertised prices while Table 5 contains the means of the advertised prices. Table 6 contains the percent discount of the advertised from the unadvertised price. This was calculated by using Equation 2:

$$
\text { Eq. } 2 \frac{\begin{array}{c}
\text { Unadvertised } \\
\text { Price }
\end{array}}{\text { Unadvertised Price }} \times 100=\text { Percent Discount }
$$

Cuts such as round steak, rump roast, fryers, and rib center cut pork chops were typically discounted by at least 20 percent with round steak discounted by 30 percent. Other cuts such as pork steak, ground beef, porterhouse steak, sirloin steak, and T-Bone steak also tended to be discounted more when advertised than the remaining meat items.

\section{Advertised Prices}

The Duncan's Multiple Range Test (DMRT) was used to determine if statistically significant differences existed $(\alpha=.05)$ in the means of the advertised prices in each of the nine stores for each meat item. Table 7 contains the results of this test. The stores are listed from the highest mean to the lowest mean prices. Across the rows, all means with the same letter do not have statistically significant differences from each other, while those that have statistically different averages have different letters.

Picnic roasts, loin center cut pork chops, and pork steak did not have significant differences between the price means of the stores that 
Table 4.

Unadvertised Price Means of the Selected Cuts of Meat in Cents/Pound in Each Store

\begin{tabular}{|c|c|c|c|c|c|c|c|c|c|}
\hline & $\begin{array}{c}\text { Regional } \\
\text { Chain } \\
(1)\end{array}$ & $\begin{array}{c}\text { Voluntary } \\
\text { Chain } \\
(2) \\
\end{array}$ & $\begin{array}{c}\text { Voluntary } \\
\text { Chain } \\
(3)\end{array}$ & $\begin{array}{c}\text { National } \\
\text { Chain } \\
(4)\end{array}$ & $\begin{array}{c}\text { National } \\
\text { Chain } \\
(5)\end{array}$ & $\begin{array}{c}\text { Regional } \\
\text { Chain } \\
(6)\end{array}$ & $\begin{array}{c}\text { Regional } \\
\text { Chain } \\
(7) \\
\end{array}$ & $\begin{array}{c}\text { Independent } \\
(8) \\
\end{array}$ & $\begin{array}{c}\text { Voluntary } \\
\text { Chain } \\
(9) \\
\end{array}$ \\
\hline Loin end roast & - & 154 & 162 & 151 & 175 & 164 & 172 & - & 139 \\
\hline $\begin{array}{l}\text { Rib center cut } \\
\text { pork chops }\end{array}$ & 210 & 229 & 220 & 227 & 228 & 244 & 242 & - & 204 \\
\hline Picnic & 109 & - & 109 & 98 & 87 & - & - & - & 99 \\
\hline \multicolumn{10}{|l|}{ Loin center cut } \\
\hline Spare ribs & 156 & 154 & 159 & 164 & 166 & 146 & 145 & 110 & 145 \\
\hline Pork steak & 169 & 179 & 159 & - & 154 & 164 & 170 & 165 & 132 \\
\hline Drumsticks & 112 & 119 & 117 & 127 & 129 & 118 & 117 & 114 & 113 \\
\hline Fryer & 72 & 71 & 69 & 69 & 69 & 68 & 68 & 70 & 69 \\
\hline Mixed parts & 69 & 61 & 64 & 59 & - & 79 & - & - & 63 \\
\hline \multicolumn{10}{|l|}{ Roasting } \\
\hline chicken & - & 90 & 95 & 90 & 98 & 75 & 88 & 95 & 79 \\
\hline Thighs & 105 & 109 & 111 & 113 & 115 & 108 & 116 & 108 & 109 \\
\hline Wings & 79 & 82 & 80 & 87 & 95 & 79 & 68 & 77 & 77 \\
\hline Whole cut-up & 77 & 74 & 76 & 79 & 76 & 80 & 76 & 77 & 76 \\
\hline Ground beef & 160 & 157 & 148 & 165 & 169 & 153 & 154 & 140 & 149 \\
\hline Ground chuck & 176 & 180 & 186 & 203 & 204 & 176 & 178 & 167 & 177 \\
\hline Ground round & - & 193 & 201 & 216 & 217 & 188 & 190 & - & 196 \\
\hline \multicolumn{10}{|l|}{ Porterhouse } \\
\hline steak & 384 & 369 & 360 & 340 & 364 & 377 & 372 & 353 & 371 \\
\hline Sirloin steak & 293 & 291 & 276 & 289 & 289 & 282 & 278 & 280 & 268 \\
\hline Stew & 216 & 224 & 222 & 256 & 248 & 218 & 218 & 191 & 187 \\
\hline T-Bone steak & 371 & 374 & 357 & 328 & 358 & 368 & 369 & 339 & 356 \\
\hline Rump roast & 286 & 270 & 265 & 261 & 295 & 274 & 267 & - & 266 \\
\hline Chuck roast & - & 159 & 163 & - & - & 191 & 166 & 186 & 171 \\
\hline Round steak & 264 & 264 & 257 & 338 & 287 & 249 & 251 & 240 & 252 \\
\hline
\end{tabular}


Advertised Price Means of the Selected Cuts of Meat in Cents/Pound in Each Store

\begin{tabular}{|c|c|c|c|c|c|c|c|c|c|}
\hline & $\begin{array}{c}\text { Regional } \\
\text { Chain } \\
(1)\end{array}$ & $\begin{array}{c}\text { Voluntary } \\
\text { Chain } \\
(2) \\
\end{array}$ & $\begin{array}{c}\text { Voluntary } \\
\text { Chain } \\
(3) \\
\end{array}$ & $\begin{array}{c}\text { National } \\
\text { Chain } \\
(4) \\
\end{array}$ & $\begin{array}{c}\text { National } \\
\text { Chain } \\
(5)\end{array}$ & $\begin{array}{c}\text { Regional } \\
\text { Chain } \\
(6)\end{array}$ & $\begin{array}{c}\text { Regional } \\
\text { Chain } \\
(7)\end{array}$ & $\begin{array}{c}\text { Independent } \\
(8) \\
\end{array}$ & $\begin{array}{c}\text { Voluntary } \\
\text { Chain } \\
(9) \\
\end{array}$ \\
\hline \multicolumn{6}{|l|}{ Rib center cut } & 137 & 137 & 137 & 144 \\
\hline pork chops & - & 159 & 159 & 192 & 192 & - & - & - & \multirow{2}{*}{$\begin{array}{l}181 \\
109\end{array}$} \\
\hline Picnic & - & - & - & 85 & 83 & - & - & - & \\
\hline & - & Loin center cut & - & 199 & 199 & 179 & - & - & 188 \\
\hline Spare ribs & 144 & 143 & 143 & 134 & 134 & 143 & 143 & 119 & 149 \\
\hline Pork steak & - & - & - & 119 & 124 & 135 & 135 & 136 & 147 \\
\hline Drumsticks & 99 & 74 & 74 & 111 & 111 & 99 & 99 & 87 & 104 \\
\hline Fryer & 48 & 57 & 51 & 49 & 49 & 62 & 62 & 55 & 56 \\
\hline Mixed parts & 54 & 57 & 57 & 52 & 52 & 49 & 49 & - & 50 \\
\hline \multicolumn{10}{|l|}{ Roasting } \\
\hline chicken & 64 & 76 & 76 & 79 & 79 & 68 & 68 & - & 60 \\
\hline Thighs & 99 & 99 & 99 & 95 & 95 & - & - & 93 & 96 \\
\hline Wings & - & 69 & 69 & 71 & 71 & - & - & 59 & 79 \\
\hline Whole cut-up & - & 66 & 66 & 64 & 64 & 109 & 109 & 52 & 65 \\
\hline Ground beef & 127 & 144 & 144 & 146 & 146 & 129 & 129 & 134 & 150 \\
\hline Ground chuck & 164 & 159 & 159 & - & - & 175 & 176 & 162 & 173 \\
\hline Ground round & - & - & - & - & - & 177 & 177 & 189 & 189 \\
\hline \multicolumn{10}{|l|}{ Porterhouse } \\
\hline steak & 286 & - & - & 305 & 296 & 299 & 299 & - & 325 \\
\hline Sirloin steak & 199 & 249 & 256 & - & - & 234 & 235 & - & 240 \\
\hline Stew & 186 & 198 & 198 & - & - & 207 & 207 & 172 & 190 \\
\hline T-Bone steak & 277 & 289 & 289 & 286 & 282 & 288 & 288 & - & 314 \\
\hline Rump roast & 222 & 194 & 200 & - & 309 & 207 & 207 & 196 & 207 \\
\hline Chuck roast & 127 & - & 169 & - & - & 118 & 118 & - & 152 \\
\hline Round steak & 181 & 167 & 167 & 182 & 182 & 189 & 189 & 192 & 204 \\
\hline
\end{tabular}


Table 6.

Percent Discount From the Unadvertised Price for All Selected Meat Cuts in Each Store

\begin{tabular}{|c|c|c|c|c|c|c|c|c|c|}
\hline y & $\begin{array}{c}\text { Regional } \\
\text { Chain } \\
(1)\end{array}$ & $\begin{array}{c}\text { Voluntary } \\
\text { Chain } \\
(2) \\
\end{array}$ & $\begin{array}{c}\text { Voluntary } \\
\text { Chain } \\
(3)\end{array}$ & $\begin{array}{c}\text { National } \\
\text { Chain } \\
(4) \\
\end{array}$ & $\begin{array}{c}\text { National } \\
\text { Chain } \\
(5) \\
\end{array}$ & $\begin{array}{c}\text { Regional } \\
\text { Chain } \\
(6) \\
\end{array}$ & $\begin{array}{c}\text { Regional } \\
\text { Chain } \\
(7) \\
\end{array}$ & $\begin{array}{c}\text { Independent } \\
(8) \\
\end{array}$ & $\begin{array}{c}\text { Voluntary } \\
\text { Chain } \\
(9) \\
\end{array}$ \\
\hline $\begin{array}{l}\text { Loin end roast } \\
\text { Rib center cut }\end{array}$ & - & 12 & 16 & - & - & 16 & 20 & - & - \\
\hline $\begin{array}{l}\text { Rib center cut } \\
\text { pork chops }\end{array}$ & - & 31 & 28 & 15 & 16 & - & - & - & 11 \\
\hline $\begin{array}{l}\text { Picnic } \\
\text { Loin center cut }\end{array}$ & - & - & - & 13 & 6 & - & - & - & - \\
\hline pork chops & - & - & - & 16 & 19 & 29 & - & - & 13 \\
\hline Spare ribs & 8 & 7 & 10 & 18 & 19 & 2 & 1 & - & - \\
\hline Pork steak & - & - & - & - & 19 & 18 & 21 & 18 & - \\
\hline Drumsticks & 11 & 38 & 37 & 13 & 14 & 16 & 15 & 24 & 8 \\
\hline Fryer & 33 & 20 & 26 & 29 & 29 & 9 & 9 & 21 & 19 \\
\hline Mixed parts & 22 & 7 & 11 & 12 & - & 38 & - & - & 21 \\
\hline Roasting & & & & & & & & & \\
\hline chicken & - & 16 & 20 & 12 & 19 & 9 & 23 & - & 24 \\
\hline Thighs & 6 & 9 & 11 & 16 & 17 & - & - & 14 & 12 \\
\hline Wings & - & 16 & 14 & 18 & 25 & - & - & 23 & - \\
\hline Whole cut-up & - & 11 & 13 & 19 & 16 & - & - & 32 & 14 \\
\hline Ground beef & 21 & 8 & 3 & 12 & 14 & 16 & 16 & 4 & - \\
\hline Ground chuck & 7 & 12 & 15 & - & - & 1 & 1 & 3 & - \\
\hline Ground round & - & - & - & - & - & 6 & 7 & - & 4 \\
\hline $\begin{array}{l}\text { Porterhouse } \\
\text { steak }\end{array}$ & 26 & - & - & 10 & 19 & 21 & 20 & - & 12 \\
\hline Sirloin steak & 32 & 14 & 7 & - & - & 17 & 15 & - & 10 \\
\hline Stew & 14 & 12 & 11 & - & - & 5 & 5 & 10 & - \\
\hline T-Bone steak & 25 & 24 & 19 & 13 & 21 & 16 & 22 & - & 12 \\
\hline Rump roast & 22 & 28 & 25 & - & - & 24 & 22 & - & 22 \\
\hline $\begin{array}{l}\text { Chuck roast } \\
\text { Round steak }\end{array}$ & $\overline{24}$ & $\overline{37}$ & $\overline{35}$ & $\overline{46}$ & $\overline{37}$ & $\begin{array}{l}38 \\
24\end{array}$ & $\begin{array}{l}29 \\
25\end{array}$ & $\overline{20}$ & $\begin{array}{l}11 \\
19\end{array}$ \\
\hline
\end{tabular}


Table 7.

Duncan's Multiple Range Test of Advertised Meat Items*

\begin{tabular}{|c|c|c|c|c|c|c|c|}
\hline Meat Item & & & & & tore & & \\
\hline Loin end roast & $\begin{array}{l}9 \\
\mathrm{~A}\end{array}$ & $\begin{array}{l}6 \\
\mathrm{~A}\end{array}$ & $\begin{array}{l}7 \\
\mathrm{~A}\end{array}$ & $\begin{array}{l}8 \\
\text { A }\end{array}$ & $\begin{array}{l}2 \\
\mathrm{~A}\end{array}$ & $\begin{array}{l}3 \\
\mathrm{~A}\end{array}$ & $\begin{array}{l}1 \\
\text { B }\end{array}$ \\
\hline Picnic & $\begin{array}{l}9 \\
\mathrm{~A}\end{array}$ & $\begin{array}{l}4 \\
\mathrm{~A}\end{array}$ & $\begin{array}{l}5 \\
\mathrm{~A}\end{array}$ & & & & \\
\hline $\begin{array}{l}\text { Rib center cut } \\
\text { pork chops }\end{array}$ & $\begin{array}{l}4 \\
\mathrm{~A}\end{array}$ & $\begin{array}{l}5 \\
\text { A }\end{array}$ & $\begin{array}{l}9 \\
\text { A } \\
\text { B }\end{array}$ & 2 & 3 & & \\
\hline
\end{tabular}

Loin center cut

pork chops

A A A A

$\begin{array}{lllllllll}9 & 1 & 2 & 3 & 6 & 7 & 4 & 5 & 8\end{array}$

Spare ribs

A A A A A A A A

B $B$ B $B$ B $B$ B $B$

Pork steak

$\begin{array}{llllll}9 & 8 & 6 & 7 & 5 & 4\end{array}$

\begin{tabular}{|c|c|c|c|c|c|c|c|c|}
\hline Drumsticks & $\begin{array}{l}4 \\
\mathrm{~A}\end{array}$ & $\begin{array}{l}5 \\
\mathrm{~A}\end{array}$ & $\begin{array}{l}9 \\
\mathrm{~A}\end{array}$ & $\begin{array}{l}1 \\
\mathrm{~A}\end{array}$ & $\begin{array}{l}6 \\
\mathrm{~A}\end{array}$ & $\begin{array}{l}7 \\
\mathrm{~A}\end{array}$ & $\begin{array}{l}8 \\
\text { A }\end{array}$ & $\begin{array}{l}2 \\
\text { A }\end{array}$ \\
\hline Fryer & $\begin{array}{l}6 \\
\mathrm{~A}\end{array}$ & $\begin{array}{l}7 \\
\mathrm{~A}\end{array}$ & $\begin{array}{l}2 \\
\mathrm{~A}\end{array}$ & $\begin{array}{l}9 \\
\mathrm{~A}\end{array}$ & $\begin{array}{l}8 \\
\mathrm{~A}\end{array}$ & $\begin{array}{l}3 \\
\mathrm{~A}\end{array}$ & $\begin{array}{l}4 \\
\mathrm{~A}\end{array}$ & $\begin{array}{l}5 \\
\mathrm{~A}\end{array}$ \\
\hline Mixed parts & $\begin{array}{l}2 \\
\mathrm{~A}\end{array}$ & $\begin{array}{l}3 \\
\mathrm{~A}\end{array}$ & $\begin{array}{l}1 \\
\mathrm{~A} \\
\mathrm{~B}\end{array}$ & $\begin{array}{l}4 \\
\text { B }\end{array}$ & $\begin{array}{l}5 \\
\text { B }\end{array}$ & $\begin{array}{l}9 \\
\text { B }\end{array}$ & $\begin{array}{l}6 \\
\text { B }\end{array}$ & 7 \\
\hline Roasting chicken & $\begin{array}{l}4 \\
\mathrm{~A}\end{array}$ & $\begin{array}{l}5 \\
\mathrm{~A}\end{array}$ & $\begin{array}{l}2 \\
\mathrm{~A}\end{array}$ & $\begin{array}{c}3 \\
\mathrm{~A}\end{array}$ & $\begin{array}{r}6 \\
\mathrm{~A}\end{array}$ & $\begin{array}{l}7 \\
\mathrm{~A}\end{array}$ & $\begin{array}{l}1 \\
\mathrm{~A}\end{array}$ & $\begin{array}{l}9 \\
\mathrm{~A}\end{array}$ \\
\hline Thighs & $\begin{array}{l}1 \\
\mathrm{~A}\end{array}$ & $\begin{array}{l}2 \\
\mathrm{~A}\end{array}$ & $\begin{array}{l}3 \\
\mathrm{~A}\end{array}$ & $\begin{array}{l}9 \\
\mathrm{~A}\end{array}$ & $\begin{array}{l}4 \\
\mathrm{~A}\end{array}$ & $\begin{array}{l}5 \\
\mathrm{~A}\end{array}$ & $\begin{array}{l}8 \\
\text { A }\end{array}$ & \\
\hline Wings & $\begin{array}{l}9 \\
\mathrm{~A}\end{array}$ & $\begin{array}{l}4 \\
\mathrm{~A} \\
\mathrm{~B}\end{array}$ & $\begin{array}{l}5 \\
\mathrm{~A} \\
\mathrm{~B}\end{array}$ & $\begin{array}{l}2 \\
\mathrm{~A} \\
\mathrm{~B}\end{array}$ & $\begin{array}{l}3 \\
\mathrm{~A} \\
\mathrm{~B}\end{array}$ & $\begin{array}{l}8 \\
\text { B }\end{array}$ & & \\
\hline Whole cut-up & $\begin{array}{l}6 \\
\mathrm{~A}\end{array}$ & $\begin{array}{l}7 \\
\text { A }\end{array}$ & $\begin{array}{l}2 \\
\text { B }\end{array}$ & $\begin{array}{l}9 \\
\text { B }\end{array}$ & $\begin{array}{l}3 \\
\text { B }\end{array}$ & $\begin{array}{l}4 \\
\text { B }\end{array}$ & $\mathrm{B}$ & C \\
\hline
\end{tabular}


Table 7 continued.

\begin{tabular}{|c|c|c|c|c|c|c|c|c|c|}
\hline Meat Item & & & & & tore & & & & \\
\hline Chuck roast & $\begin{array}{l}3 \\
\mathrm{~A}\end{array}$ & $\begin{array}{l}9 \\
\mathrm{~A}\end{array}$ & $\begin{array}{l}1 \\
\mathrm{~A}\end{array}$ & $\begin{array}{l}6 \\
\mathrm{~A}\end{array}$ & $\begin{array}{l}7 \\
\mathrm{~A}\end{array}$ & & & & \\
\hline Ground beef & $\begin{array}{l}9 \\
\mathrm{~A}\end{array}$ & $\begin{array}{l}4 \\
\mathrm{~A}\end{array}$ & $\begin{array}{l}5 \\
\mathrm{~A}\end{array}$ & $\begin{array}{l}2 \\
\mathrm{~A} \\
\mathrm{~B}\end{array}$ & $\begin{array}{l}3 \\
\mathrm{~A} \\
\mathrm{~B}\end{array}$ & $\begin{array}{l}8 \\
\text { B }\end{array}$ & $\begin{array}{l}6 \\
B\end{array}$ & $\begin{array}{l}7 \\
\text { B }\end{array}$ & $\begin{array}{l}1 \\
B\end{array}$ \\
\hline Ground chuck & $\begin{array}{l}7 \\
\mathrm{~A}\end{array}$ & $\begin{array}{l}6 \\
\mathrm{~A}\end{array}$ & $\begin{array}{l}9 \\
\mathrm{~A}\end{array}$ & $\begin{array}{l}1 \\
\mathrm{~B}\end{array}$ & $\begin{array}{l}8 \\
\text { B }\end{array}$ & $\begin{array}{l}2 \\
\text { B }\end{array}$ & $\begin{array}{l}3 \\
\text { B }\end{array}$ & & \\
\hline Ground round & $\begin{array}{l}8 \\
\mathrm{~A}\end{array}$ & $\begin{array}{l}9 \\
\mathrm{~A} \\
\mathrm{~B}\end{array}$ & $\begin{array}{l}6 \\
\text { B }\end{array}$ & $\begin{array}{l}7 \\
\text { B }\end{array}$ & & & & & \\
\hline $\begin{array}{l}\text { Porterhouse } \\
\text { steak }\end{array}$ & $\begin{array}{l}9 \\
\text { A }\end{array}$ & $\begin{array}{l}4 \\
\text { B }\end{array}$ & $\begin{array}{l}6 \\
\text { B }\end{array}$ & $\begin{array}{l}7 \\
\text { B }\end{array}$ & $\begin{array}{l}5 \\
\text { B }\end{array}$ & $\begin{array}{l}1 \\
\text { B }\end{array}$ & & & \\
\hline Round steak & $\begin{array}{l}9 \\
\mathrm{~A}\end{array}$ & $\begin{array}{l}8 \\
\text { A } \\
\text { B }\end{array}$ & $\begin{array}{l}6 \\
\text { B }\end{array}$ & $\begin{array}{l}7 \\
\text { B }\end{array}$ & $\begin{array}{l}4 \\
\text { B }\end{array}$ & $\begin{array}{l}5 \\
\text { B }\end{array}$ & $\begin{array}{l}1 \\
\text { B } \\
\text { C }\end{array}$ & $\begin{array}{l}2 \\
\text { C }\end{array}$ & $\begin{array}{l}3 \\
\mathrm{C}\end{array}$ \\
\hline Rump roast & $\begin{array}{l}5 \\
\mathrm{~A}\end{array}$ & $\begin{array}{l}1 \\
\text { B }\end{array}$ & $\begin{array}{l}6 \\
\mathrm{~B} \\
\mathrm{C}\end{array}$ & $\begin{array}{c}7 \\
\text { B } \\
\text { C }\end{array}$ & 9 & 3 & 8 & 2 & \\
\hline Sirloin steak & $\begin{array}{l}3 \\
\mathrm{~A}\end{array}$ & $\begin{array}{l}2 \\
\text { A } \\
B\end{array}$ & $\begin{array}{l}9 \\
\text { A } \\
\text { B }\end{array}$ & $\begin{array}{c}6 \\
\mathrm{~A} \\
\mathrm{~B}\end{array}$ & $\begin{array}{l}7 \\
\text { A } \\
\text { B }\end{array}$ & $\begin{array}{l}1 \\
\mathrm{~B}\end{array}$ & & & \\
\hline Stew & $\begin{array}{l}6 \\
\mathrm{~A}\end{array}$ & $\begin{array}{l}7 \\
\mathrm{~A}\end{array}$ & $\begin{array}{l}2 \\
\text { B }\end{array}$ & $\begin{array}{l}3 \\
B\end{array}$ & $\begin{array}{l}9 \\
\mathrm{~B} \\
\mathrm{C}\end{array}$ & 1 & 8 & & \\
\hline T-Bone steak & $\begin{array}{l}9 \\
\mathrm{~A}\end{array}$ & $\begin{array}{l}2 \\
\mathrm{~A} \\
\mathrm{~B}\end{array}$ & $\begin{array}{l}3 \\
\mathrm{~A} \\
\mathrm{~B}\end{array}$ & $\begin{array}{l}6 \\
\mathrm{~A} \\
\mathrm{~B}\end{array}$ & $\begin{array}{l}7 \\
\mathrm{~A} \\
\mathrm{~B}\end{array}$ & $\begin{array}{l}4 \\
\text { B }\end{array}$ & $\begin{array}{l}5 \\
\text { B }\end{array}$ & $\begin{array}{l}1 \\
\mathrm{~B}\end{array}$ & \\
\hline
\end{tabular}

*Those means,with the same letter are not significantly different at the $\alpha=.05$ leve The stores' means are rankęd from highest to lowest. 
advertised. There were some statistically significant differences between the average advertised prices for the other pork cuts. It is also noted that Store 9, a voluntary chain, tended to have higher average prices than the other stores. However, the average prices for Store 9 were not statistically significantly different from those of the other stores. Similarly, Store 1 tended to have lower advertised prices than the other stores.

Four of the seven chicken cuts (drumsticks, fryers, roasting chickens, and thighs) had significantly different prices between stores. Store 8, the independent, had a significantly lower mean price for whole cut-up chicken and there were some significant differences between the mean prices for the other cuts of chicken.

There appear to be more differences among the stores for the average prices of the various cuts of beef than for pork or chicken. However, except for a tendency toward lower prices in Store 1 and higher prices in Store 9 no distinct patterns were apparent. Chuck roast was the only cut for which there were statistically significant differences between the averages of the stores that advertised it. Store 9 did tend to have average beef prices near the upper end of the ranges of prices.

\section{Unadvertised Prices}

Table 8 contains the results of the Duncan's Test for the means of unadvertised meat items in each store. The means for the pork cuts do not appear to be very different. Store 9, a voluntary chain, appears in the lower range of means for most of the cuts of pork. As shown in Table 7 , this store tended to have higher advertised prices, but the unadvertised prices tended to be lower than the other stores.

Whether a chicken fryer is advertised or unadvertised the mean prices between the stores generally are not significantly different. For mixed parts and whole cut-up chickens, there were no significant differences between the means of unadvertised prices. There were significant differences between the means for the other cuts of chicken in some of the stores but no definite patterns were observed. Store 5 tended to have somewhat lower unadvertised chicken prices while Store 9 tended to have higher prices.

There seemed to be considerable differences between averages for the unadvertised prices of ground beef and ground round. The national chain had higher unadvertised prices for ground beef and also was among the stores with higher prices for ground round. Furthermore, the national chain appeared to be higher in the range of unadvertised mean prices for six of the ten cuts of beef. Stores 8 and 9 tended to have lower unadvertised prices for beef while Stores 1, 4, 


\section{Table 8.}

Duncan's Multiple Range Test of Unadvertised Meat Items in Each Store*

\begin{tabular}{|c|c|c|c|c|c|c|c|c|}
\hline Meat Item & & & & & tor & & & \\
\hline Loin end roast & $\begin{array}{l}5 \\
\mathrm{~A}\end{array}$ & $\begin{array}{l}7 \\
\mathrm{~A}\end{array}$ & $\begin{array}{l}6 \\
\mathrm{~A}\end{array}$ & $\begin{array}{l}3 \\
\mathrm{~A}\end{array}$ & $\begin{array}{l}2 \\
\mathrm{~A}\end{array}$ & $\begin{array}{l}4 \\
\mathrm{~A}\end{array}$ & $\begin{array}{l}9 \\
\mathrm{~A}\end{array}$ & \\
\hline Picnic & $\begin{array}{l}1 \\
\mathrm{~A}\end{array}$ & $\begin{array}{l}3 \\
\mathrm{~A}\end{array}$ & $\begin{array}{l}9 \\
\mathrm{~A} \\
\mathrm{~B}\end{array}$ & $\begin{array}{l}4 \\
\text { B }\end{array}$ & $\begin{array}{l}5 \\
\mathrm{~B}\end{array}$ & & & \\
\hline $\begin{array}{l}\text { Rib center cut } \\
\text { pork chops }\end{array}$ & $\begin{array}{l}6 \\
\text { A }\end{array}$ & $\begin{array}{c}7 \\
\mathrm{~A}\end{array}$ & $\begin{array}{l}2 \\
\text { A } \\
\text { B }\end{array}$ & 5 & 4 & $\begin{array}{l}\mathrm{B} \\
\mathrm{C}\end{array}$ & 1 & 9 \\
\hline
\end{tabular}

Loin center cut pork chops

$\begin{array}{lllllllll}8 & 7 & 6 & 5 & 4 & 2 & 3 & 1 & 9\end{array}$

A A A A A A A

B $\quad$ B $\quad B \quad B$

Spare ribs $\begin{array}{lllllllll}5 & 4 & 3 & 1 & 2 & 6 & 9 & 7 & 8\end{array}$ A A A A A

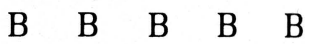

Pork steak

$\begin{array}{llllllll}2 & 7 & 1 & 8 & 6 & 3 & 5 & 9\end{array}$
A A A A A A A B $B$ B $\quad$ B $\quad$ B $\quad$ B $\begin{array}{lllllllll}5 & 4 & 2 & 6 & 3 & 7 & 8 & 9 & 1\end{array}$ Drumsticks A A

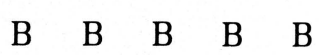
C $\quad$ C $\quad$ C $\begin{array}{lllllllll}1 & 2 & 8 & 5 & 9 & 4 & 3 & 6 & 7\end{array}$

Fryer A A A A A A A A A

Mixed parts $\begin{array}{llllll}6 & 1 & 3 & 9 & 2 & 4\end{array}$ A A A A A A

Roasting chicken $\begin{array}{llllllll}5 & 8 & 3 & 2 & 4 & 7 & 9 & 6\end{array}$ A A A A A A A 
Table 8 continued.

\section{Meat Item}

\section{Store}

Thighs

$\begin{array}{lllllllll}7 & 5 & 4 & 3 & 9 & 2 & 6 & 8 & 1\end{array}$

A A A

B $B \quad$ B

C $\quad \mathrm{C} \quad \mathrm{C} \quad \mathrm{C} \quad \mathrm{C} \quad \mathrm{C}$

D D D D D

$\begin{array}{lllllllll}5 & 4 & 2 & 3 & 1 & 6 & 8 & 9 & 7\end{array}$

Wings

$\begin{array}{clllllll}\text { A } & \text { A } & & & & & & \\ & \text { B } & \text { B } & \text { B } & \text { B } & \text { B } & \text { B } & \text { B }\end{array}$

C $\mathrm{C} \quad \mathrm{C} \quad \mathrm{C}$ C

$\begin{array}{lllllllll}6 & 4 & 8 & 1 & 5 & 7 & 3 & 9 & 2\end{array}$

Whole cut-up

A A A A A A A A A

Chuck roast

$\begin{array}{llllll}6 & 8 & 9 & 7 & 3 & 2\end{array}$

A A

B $B$ B B $B$

$\begin{array}{lllllllll}5 & 4 & 1 & 2 & 7 & 6 & 9 & 3 & 8\end{array}$

Ground beef A A

$$
\text { B B }
$$

C C C

D D D

E E E

F F F

Ground chuck

$\begin{array}{lllllllll}5 & 4 & 3 & 2 & 7 & 9 & 6 & 1 & 8\end{array}$

A A

B

$\begin{array}{lllllllllllllll}C & \mathrm{C} & \mathrm{C} & \mathrm{C} & \mathrm{C}\end{array}$

D

$\begin{array}{lllllll}5 & 4 & 3 & 9 & 2 & 7 & 6\end{array}$

Ground round

A A

B

$$
\begin{array}{llll}
C & C & & \\
& D & D & \\
& E & E
\end{array}
$$


Table 8 continued.

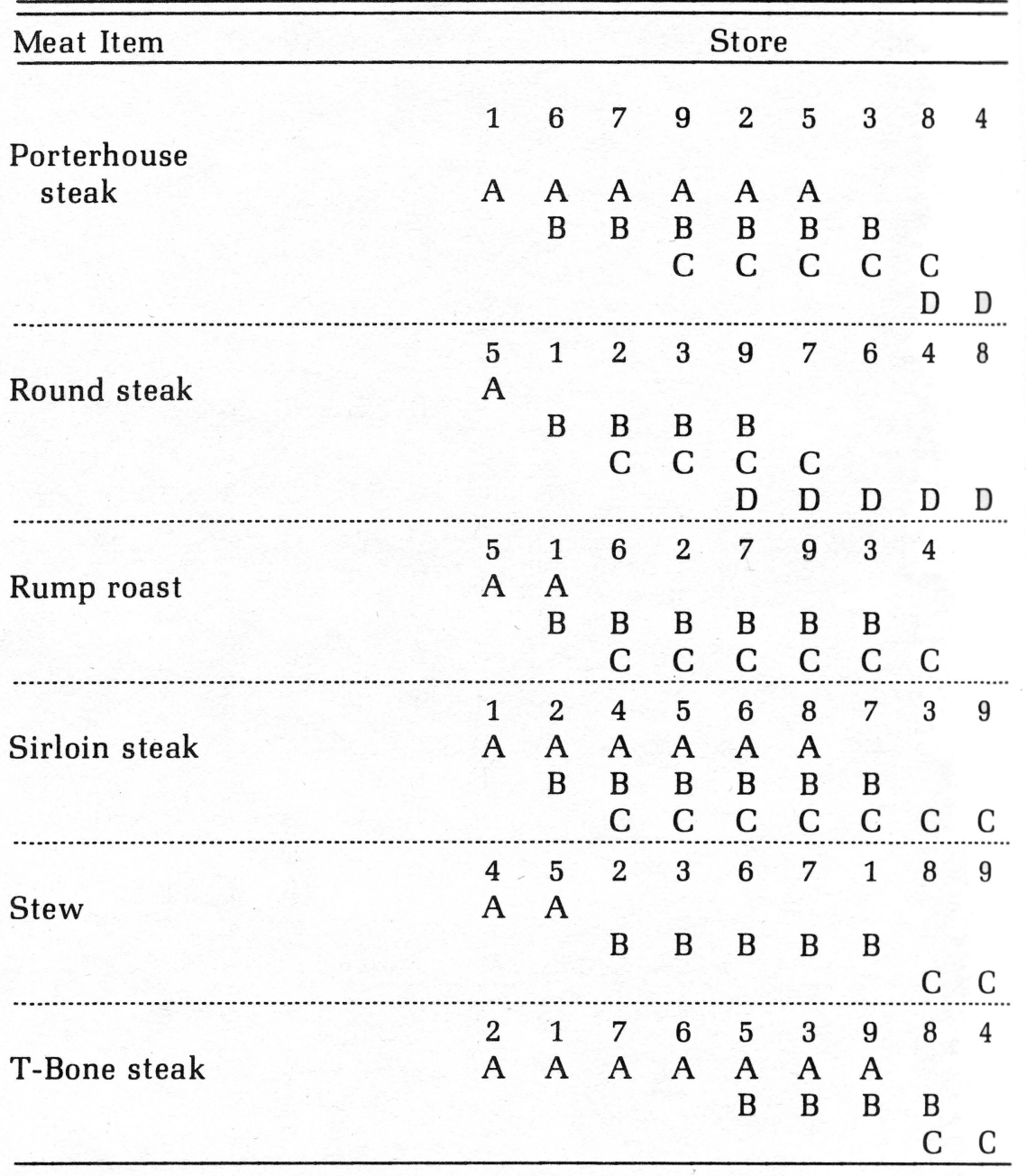

* Those means with the same letter are not significantly different at the $\alpha=.05$ level. The stores' means are ranked from highest to lowest.

and 5 tended to have lower prices, that is, tended to have prices in the lower one-third of the range of prices.

\section{All Prices}

Table 9 contains the mean prices of each meat item for each store regardless of whether the meat item was advertised or unadvertised. 
Table 9.

Mean Prices of Each Meat Item, for Each Store Regardless of Whether the Meat Item is Advertised or Unadvertised (Cents/Pound)

\begin{tabular}{|c|c|c|c|c|c|c|c|c|c|}
\hline & $\begin{array}{c}\text { Regional } \\
\text { Chain } \\
(1)\end{array}$ & $\begin{array}{c}\text { Voluntary } \\
\text { Chain } \\
(2)\end{array}$ & $\begin{array}{c}\text { Voluntary } \\
\text { Chain } \\
(3)\end{array}$ & $\begin{array}{c}\text { National } \\
\text { Chain } \\
(4)\end{array}$ & $\begin{array}{c}\text { National } \\
\text { Chain } \\
(5)\end{array}$ & $\begin{array}{c}\text { Regional } \\
\text { Chain } \\
(6)\end{array}$ & $\begin{array}{c}\text { Regional } \\
\text { Chain } \\
(7)\end{array}$ & $\begin{array}{c}\text { Independent } \\
(8) \\
\end{array}$ & $\begin{array}{c}\text { Voluntary } \\
\text { Chain } \\
(9)\end{array}$ \\
\hline Loin end roast & 109 & 151 & \multicolumn{7}{|c|}{ Rib center cut } \\
\hline pork chops & 210 & 220 & 218 & 222 & 224 & 244 & 242 & - & 188 \\
\hline Picnic & 109 & - & 109 & 92 & 84 & - & - & - & 102 \\
\hline \multicolumn{10}{|l|}{ Loin center cut } \\
\hline pork chops & 217 & 227 & 227 & 235 & 243 & 249 & 252 & 269 & 213 \\
\hline Spare ribs & 149 & 148 & 155 & 161 & 163 & 145 & 144 & 111 & 147 \\
\hline Pork steak & 169 & 179 & 159 & 119 & 139 & 159 & 163 & 161 & 139 \\
\hline Drumsticks & 111 & 116 & 114 & 126 & 218 & 117 & 116 & 105 & 110 \\
\hline Fryer & 71 & 69 & 67 & 62 & 62 & 68 & 68 & 66 & 65 \\
\hline Mixed parts & 68 & 60 & 62 & 52 & 52 & 64 & 49 & - & 56 \\
\hline \multicolumn{10}{|l|}{ Roasting } \\
\hline chicken & 64 & 89 & 92 & 90 & 97 & 74 & 86 & 95 & 65 \\
\hline Thighs & 104 & 108 & 110 & 108 & 109 & 108 & 116 & 104 & 106 \\
\hline Wings & 79 & 81 & 79 & 86 & 94 & 79 & 68 & 71 & 78 \\
\hline Whole cut-up & 77 & 72 & 73 & 75 & 73 & 81 & 77 & 73 & 73 \\
\hline
\end{tabular}


Table 9 continued.

\begin{tabular}{|c|c|c|c|c|c|c|c|c|c|}
\hline Meat Item & $\begin{array}{c}\text { Regional } \\
\text { Chain } \\
(1)\end{array}$ & $\begin{array}{c}\text { Voluntary } \\
\text { Chain } \\
(2) \\
\end{array}$ & $\begin{array}{c}\text { Voluntary } \\
\text { Chain } \\
(3) \\
\end{array}$ & $\begin{array}{c}\text { National } \\
\text { Chain } \\
(4) \\
\end{array}$ & $\begin{array}{c}\text { National } \\
\text { Chain } \\
(5) \\
\end{array}$ & $\begin{array}{c}\text { Regional } \\
\text { Chain } \\
(6)\end{array}$ & $\begin{array}{c}\text { Regional } \\
\text { Chain } \\
(7)\end{array}$ & $\begin{array}{c}\text { Independent } \\
(8) \\
\end{array}$ & $\begin{array}{c}\text { Voluntary } \\
\text { Chain } \\
(9) \\
\end{array}$ \\
\hline Ground beef & 158 & 156 & 147 & 156 & 158 & 152 & 153 & 136 & 149 \\
\hline Ground chuck & 175 & 177 & 182 & 203 & 204 & 176 & 178 & 164 & 175 \\
\hline Ground round & - & 193 & 201 & 216 & 217 & 186 & 189 & 189 & 196 \\
\hline $\begin{array}{l}\text { Porterhouse } \\
\text { steak }\end{array}$ & 365 & 369 & 360 & 320 & 337 & 374 & 369 & 353 & 356 \\
\hline Sirloin steak & 271 & 288 & 274 & 289 & 289 & 274 & 276 & 280 & 256 \\
\hline Stew & 208 & 217 & 216 & 256 & 248 & 214 & 214 & 178 & 188 \\
\hline T-Bone steak & 349 & 370 & 354 & 316 & 341 & 365 & 366 & 339 & 342 \\
\hline Rump roast & 264 & 238 & 242 & 261 & 296 & 258 & 253 & 196 & 238 \\
\hline Chuck roast & 127 & 159 & 163 & - & - & 185 & 163 & 186 & 167 \\
\hline Round steak & 256 & 238 & 233 & 214 & 244 & 238 & 239 & 232 & 225 \\
\hline
\end{tabular}


The results of the Duncan's Test used to determine if significant differences existed are shown in Table 10. For the different cuts of chicken, pork, and beef, some statistically significant differences between the mean prices were found. Stores 8 and 9 , one a voluntary chain and the other an independent, seem to have had lower average prices for most cuts of beef, while Stores 4 and 5 tended to somewhat higher prices.

Table 10.

Duncan's Multiple Range Test for the Means of the Selected Meat Items Regardless of Whether It Is Advertised or Unadvertised*

\begin{tabular}{|c|c|c|c|c|c|c|c|c|c|}
\hline Meat Item & & & & & tor & & & & \\
\hline Loin end roast & $\begin{array}{l}5 \\
\mathrm{~A}\end{array}$ & $\begin{array}{l}7 \\
\mathrm{~A}\end{array}$ & $\begin{array}{l}6 \\
A\end{array}$ & $\begin{array}{l}3 \\
\mathrm{~A}\end{array}$ & $\begin{array}{l}4 \\
\mathrm{~A}\end{array}$ & $\begin{array}{l}2 \\
\mathrm{~A}\end{array}$ & $\begin{array}{l}9 \\
\mathrm{~A}\end{array}$ & $\begin{array}{l}8 \\
A\end{array}$ & 1 \\
\hline Picnic & $\begin{array}{l}1 \\
\mathrm{~A}\end{array}$ & $\begin{array}{l}3 \\
\mathrm{~A}\end{array}$ & $\begin{array}{l}9 \\
\text { A } \\
\text { B }\end{array}$ & 4 & 5 & & & & \\
\hline
\end{tabular}

\begin{tabular}{|c|c|c|c|c|c|c|c|c|}
\hline & \multicolumn{8}{|c|}{ C } \\
\hline & 6 & 7 & 5 & 4 & 2 & 3 & 1 & 9 \\
\hline Rib center cut & A & A & & & & & & \\
\hline pork chops & & & B & B & B & B & & \\
\hline
\end{tabular}

Loin center cut pork chops

Spare ribs $\begin{array}{lllllllll}8 & 7 & 6 & 5 & 4 & 2 & 3 & 1 & 9\end{array}$ A A A A A A A

$\begin{array}{lllllllll}5 & 4 & 3 & 1 & 2 & 9 & 6 & 7 & 8\end{array}$

A A A

$$
\begin{array}{lllllll}
\text { B } & \text { B } & \text { B } & \text { B } & & & \\
& \text { C } & \text { C } & \text { C } & \text { C } & & \\
& & \text { D } & \text { D } & \text { D } & \text { D } & \text { D }
\end{array}
$$

Pork steak

$\begin{array}{lllllllll}2 & 1 & 7 & 8 & 3 & 6 & 5 & 9 & 4\end{array}$

A A A A A A A A

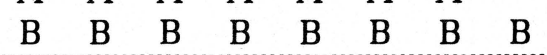


Table 10 continued.

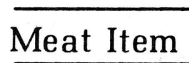

Store

Drumsticks

$\begin{array}{lllllllll}5 & 4 & 6 & 2 & 7 & 3 & 1 & 9 & 8 \\ \mathrm{~A} & \mathrm{~A} & & & & & & & \end{array}$ $\begin{array}{lllll}\text { B } & \text { B } & \text { B } & \text { B } & \\ & \text { C } & \text { C } & \text { C } & \text { C }\end{array}$ D D D

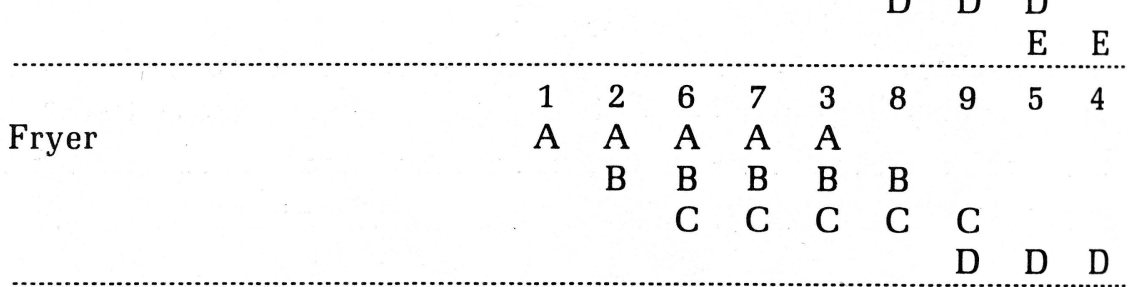

Mixed parts

$\begin{array}{llllllll}1 & 6 & 3 & 2 & 9 & 4 & 5 & 7\end{array}$

A A

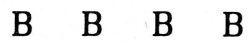

C C C

Roasting chicken

$\begin{array}{lllllllll}5 & 8 & 3 & 4 & 2 & 7 & 6 & 9 & 1\end{array}$

A A A A A

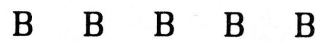

Thighs

$\begin{array}{lllllllll}7 & 3 & 5 & 2 & 6 & 4 & 9 & 1 & 8\end{array}$

A

$\begin{array}{llllll}B & B & B & B & B & B\end{array}$

$\begin{array}{lllllll}C & C & C & C & C & C & C\end{array}$

Wings

$\begin{array}{lllllllll}5 & 4 & 2 & 1 & 6 & 3 & 9 & 8 & 7\end{array}$

A A

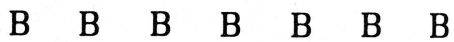

C C C C C C

Whole cut-up

$\begin{array}{lllllllll}6 & 7 & 1 & 4 & 8 & 9 & 5 & 3 & 2\end{array}$

A A A

B $B$ B B B B B

$\begin{array}{lllllll}8 & 6 & 9 & 3 & 7 & 2 & 1\end{array}$

Chuck roast

A A A A A A A

continued 
Table 10 continued.

\begin{tabular}{lcccccccccc}
\hline \hline Meat Item & 11 & \multicolumn{11}{c}{ Store } \\
\hline \multirow{3}{*}{ Ground beef } & 5 & 1 & 4 & 2 & 7 & 6 & 9 & 3 & 8 \\
& A & A & A & A & A & & & & \\
& & B & B & B & B & B & & & \\
& & & & & C & C & C & & \\
& & & & & & D & D & D &
\end{tabular}

Ground chuck

$\begin{array}{lllllllll}5 & 4 & 3 & 7 & 2 & 6 & 9 & 1 & 8\end{array}$

A A

$$
\begin{array}{lllllll}
\text { B } & \text { B } & \text { B } & & & \\
& \text { C } & \text { C } & \text { C } & \text { C } & \text { C }
\end{array}
$$

D

Ground round

$\begin{array}{llllllll}5 & 4 & 3 & 9 & 2 & 8 & 7 & 6 \\ \mathrm{~A} & \mathrm{~A} & & & \end{array}$

B

C C C

D D D

Porterhouse steak

$\begin{array}{lllllllll}6 & 7 & 2 & 1 & 3 & 9 & 8 & 5 & 4\end{array}$

A A A A A

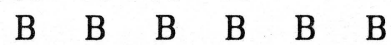

Round steak

$\begin{array}{lllllllll}1 & 5 & 7 & 6 & 2 & 3 & 8 & 9 & 4\end{array}$

A

B $\quad$ B $\quad$ B $\quad$ B

C C C C C

D D D

E E

Rump roast

$\begin{array}{lllllllll}5 & 1 & 4 & 6 & 7 & 3 & 9 & 2 & 8\end{array}$

A

$\begin{array}{lllll}\text { B } & \text { B } & \text { B } & \text { B } & \\ & \text { C } & \text { C } & \text { C } & \text { C }\end{array}$

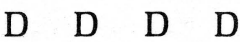

Sirloin steak

$\begin{array}{lllllllll}4 & 5 & 2 & 8 & 6 & 7 & 3 & 1 & 9\end{array}$

A A A A A A A A A

continued 
Table 10 continued.

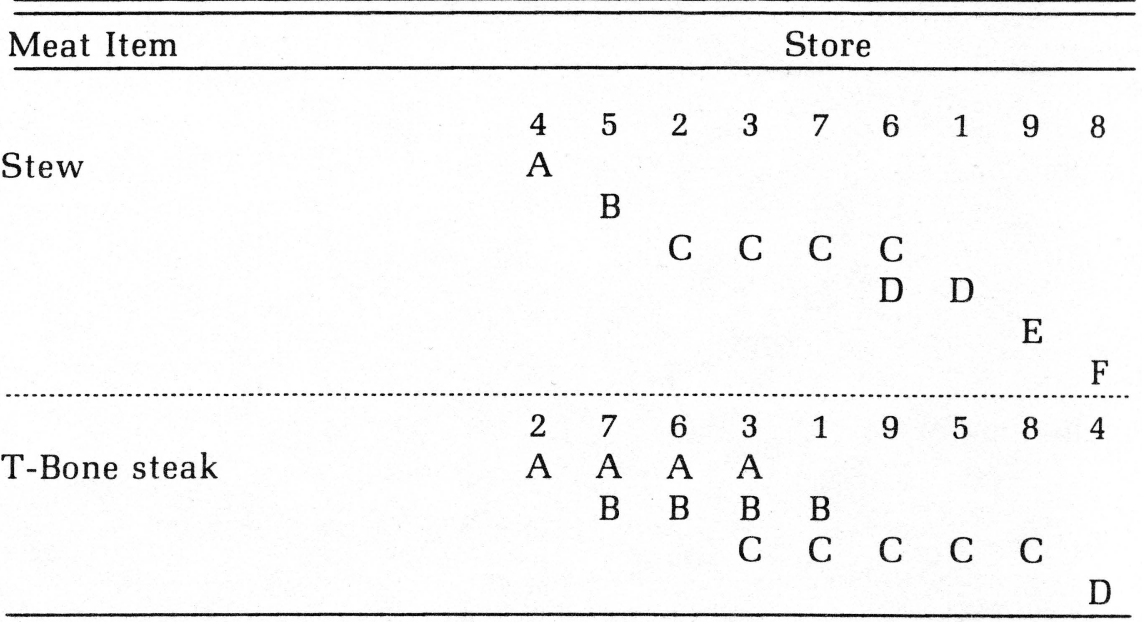

*Those means with the same letter are not significantly different at the $\alpha=.05$ level. The stores' means are ranked from highest to lowest.

Table 11 contains the advertised and the unadvertised price means regardless of store. Again, a Duncan's Test was applied. All the cuts had statistically significant differences between advertised mean prices and the unadvertised mean prices except for chicken wings. This confirms that advertised prices for cuts of meat generally are lower than the unadvertised prices. The average discounts range from 4 to 29 percent. For beef, pork, and chicken, the average discounts were 16,15 , and 18 percent, respectively.

\section{Type of Store}

The stores were grouped according to type-chain, voluntary chain, and independent. The means and frequencies for these groupings are shown in Table 12. A Duncan's Test was used to determine if there were significant differences between the mean prices for each type of store (Table 13). Loin center cut pork chops, roasting chickens, fryers, wings, ground round, porterhouse steaks, sirloin steaks, T-Bone steaks, chuck roasts, and round steaks were cuts for which there were no statistically significant differences between the store mean prices. Several cuts had differences between two store types while drumsticks, ground beef, ground chuck, and stew meat all had significantly different average prices between all three types of stores. There appear to be fewer differences between the means of the 
Table 11.

Advertised and Unadvertised Price Means (Cents/Pound) Regardless of Store and Duncan's Multiple Range Test Where $\propto=.05$

\begin{tabular}{|c|c|c|c|c|c|}
\hline Meat Item & Advertised & Unadvertised & Difference & $\begin{array}{c}\text { Percent } \\
\text { Discount }^{*} \\
\end{array}$ & $\begin{array}{c}\text { Duncan's Multiple } \\
\text { Range Test }\end{array}$ \\
\hline Loin end roast & 134 & 160 & 26 & 16 & Significantly Different \\
\hline Rib center cut pork chops & 85 & 102 & 17 & 17 & Significantly Different \\
\hline Picnic & 183 & 229 & 36 & 16 & Significantly Different \\
\hline Loin center cut pork chops & 190 & 235 & 45 & 19 & Significantly Different \\
\hline Spare ribs & 143 & 149 & 6 & 4 & Significantly Different \\
\hline Pork steak & 137 & 163 & 26 & 16 & Significantly Different \\
\hline Drumsticks & 95 & 119 & 24 & 20 & Significantly Different \\
\hline Fryer & 53 & 69 & 16 & 23 & Significantly Different \\
\hline Mixed parts & 53 & 65 & 12 & 18 & Significantly Different \\
\hline Roasting chicken & 68 & 88 & 20 & 23 & Significantly Different \\
\hline Thighs & 95 & 110 & 15 & 17 & Significantly Different \\
\hline Wings & 72 & 81 & 9 & 11 & No Significant Difference \\
\hline Whole cut-up & 66 & 77 & 11 & 14 & Significantly Different \\
\hline Ground beef & 141 & 155 & 14 & 9 & Significantly Different \\
\hline Ground chuck & 167 & 185 & 18 & 10 & Significantly Different \\
\hline Ground round & 181 & 199 & 18 & 9 & Significantly Different \\
\hline Porterhouse steak & 305 & 366 & 61 & 17 & Significantly Different \\
\hline Sirloin steak & 230 & 282 & 52 & 18 & Significantly Different \\
\hline Stew & 193 & 221 & 28 & 13 & Significantly Different \\
\hline T-Bone steak & 292 & 357 & 65 & 18 & Significantly Different \\
\hline Rump roast & 208 & 274 & 66 & 24 & Significantly Different \\
\hline Chuck roast & 145 & 172 & 27 & 16 & Significantly Different \\
\hline Round steak & 183 & 256 & 73 & 29 & Significantly Different \\
\hline
\end{tabular}

*The a verage discount for pork, chicken, and beef was 15 percent, 18 percent, and 16 percent, respectively. The average total discount was 16 percent. 


\section{Table 12.}

The Means (Cents/Pound) of Each Meat Item by Type of Store. Also the Frequency Distribution of Each Meat Item by Type of Store

\begin{tabular}{|c|c|c|c|c|c|c|}
\hline \multirow[b]{2}{*}{ Meat Items } & \multicolumn{3}{|c|}{ MEAN } & \multicolumn{3}{|c|}{ FREQUENCY } \\
\hline & Chain & Independent & $\begin{array}{c}\text { Voluntary } \\
\text { Chain }\end{array}$ & Chain & Independent & $\begin{array}{c}\text { Voluntary } \\
\text { Chain }\end{array}$ \\
\hline Loin end roast & 159 & 137 & 151 & 62 & 5 & 32 \\
\hline Rib center cut pork chops & 230 & - & 215 & 141 & - & 47 \\
\hline Picnic & 94 & - & 105 & 55 & - & 8 \\
\hline Loin center cut pork chops & 240 & 269 & 221 & 147 & 1 & 78 \\
\hline Spare ribs & 152 & 111 & 151 & 97 & 18 & 48 \\
\hline Pork steak & 159 & 161 & 145 & 57 & 19 & 17 \\
\hline Drumsticks & 120 & 105 & 114 & 148 & 16 & 96 \\
\hline Fryer & 66 & 66 & 67 & 144 & 31 & 100 \\
\hline Mixed parts & 57 & - & 59 & 97 & - & 84 \\
\hline Roasting chicken & 85 & 95 & 87 & 104 & 5 & 59 \\
\hline Thighs & 190 & 104 & 108 & 154 & 21 & 96 \\
\hline Wings & 81 & 71 & 79 & 99 & 3 & 56 \\
\hline Whole cut-up & 76 & 73 & 73 & 151 & 19 & 98 \\
\hline Ground beef & 155 & 136 & 151 & 167 & 34 & 98 \\
\hline Ground chuck & 187 & 164 & 178 & 160 & 34 & 97 \\
\hline Ground round & 199 & 189 & 197 & 112 & 3 & 96 \\
\hline Porterhouse steak & 355 & 353 & 361 & 141 & 29 & 90 \\
\hline Sirloin steak & 276 & 280 & 273 & 97 & 26 & 87 \\
\hline Stew & 225 & 178 & 206 & 140 & 23 & 98 \\
\hline T-Bone steak & 346 & 339 & 353 & 146 & 23 & 83 \\
\hline Rump roast & 265 & 196 & 239 & 115 & 3 & 76 \\
\hline Chuck roast & $\begin{array}{l}172 \\
240\end{array}$ & 186 & 165 & 31 & $\begin{array}{r}3 \\
34\end{array}$ & $\begin{array}{l}51 \\
74\end{array}$ \\
\hline
\end{tabular}


Table 13.

Duncan's Multiple Range Test on Means of the Selected Meat Items by Store Type*

\begin{tabular}{lccc}
\hline \hline & & Voluntary & \\
& Chain & Chain & Independent \\
Loin end roast & A & A & B \\
& & B &
\end{tabular}

$\begin{array}{ccc}\text { Voluntary } & \\ \text { Chain } & \text { Chain } \\ \text { Picnic } & \text { A } & \text { B }\end{array}$

\begin{tabular}{|c|c|c|c|}
\hline $\begin{array}{l}\text { Rib center cut } \\
\text { pork chops }\end{array}$ & $\begin{array}{c}\text { Chain } \\
\text { A }\end{array}$ & $\begin{array}{c}\text { Voluntary } \\
\text { Chain }\end{array}$ & \\
\hline $\begin{array}{l}\text { Loin center cut } \\
\text { pork chops }\end{array}$ & Independent & $\begin{array}{c}\text { Chain } \\
\text { A }\end{array}$ & $\begin{array}{c}\text { Voluntary } \\
\text { Chain }\end{array}$ \\
\hline Spare ribs & $\begin{array}{c}\text { Chain } \\
\text { A }\end{array}$ & $\begin{array}{l}\text { Voluntary } \\
\text { Chain } \\
\text { A }\end{array}$ & $\begin{array}{c}\text { Independent } \\
\text { B }\end{array}$ \\
\hline Pork steak & $\begin{array}{c}\text { Independent } \\
\text { A }\end{array}$ & $\begin{array}{c}\text { Chain } \\
\text { A }\end{array}$ & $\begin{array}{c}\text { Voluntary } \\
\text { Chain } \\
\text { B }\end{array}$ \\
\hline Drumsticks & $\begin{array}{c}\text { Chain } \\
\text { A }\end{array}$ & $\begin{array}{c}\text { Voluntary } \\
\text { Chain } \\
\text { B }\end{array}$ & Independent \\
\hline Fryer & $\begin{array}{c}\text { Voluntary } \\
\text { Chain } \\
\text { A }\end{array}$ & $\begin{array}{c}\text { Independent } \\
\text { A }\end{array}$ & $\begin{array}{l}\text { Chain } \\
\text { A }\end{array}$ \\
\hline
\end{tabular}


Table 13 continued.

\begin{tabular}{|c|c|c|c|}
\hline Mixed parts & $\begin{array}{c}\text { Voluntary } \\
\text { Chain } \\
\text { A }\end{array}$ & $\begin{array}{c}\text { Chain } \\
\text { B }\end{array}$ & \\
\hline Roasting chicken & Independent & $\begin{array}{c}\text { Voluntary } \\
\text { Chain } \\
\text { A }\end{array}$ & $\begin{array}{c}\text { Chain } \\
\text { A }\end{array}$ \\
\hline Thighs & $\begin{array}{c}\text { Chain } \\
\text { A }\end{array}$ & $\begin{array}{c}\text { Voluntary } \\
\text { Chain } \\
\text { A } \\
\text { B }\end{array}$ & $\begin{array}{c}\text { Independent } \\
\text { B }\end{array}$ \\
\hline Wings & $\begin{array}{c}\text { Chain } \\
\text { A }\end{array}$ & $\begin{array}{c}\text { Voluntary } \\
\text { Chain } \\
\text { A }\end{array}$ & Independent \\
\hline Whole cut-up & $\begin{array}{c}\text { Chain } \\
\text { A }\end{array}$ & $\begin{array}{c}\text { Independent } \\
\text { A } \\
\text { B }\end{array}$ & $\begin{array}{c}\text { Voluntary } \\
\text { Chain } \\
\text { B }\end{array}$ \\
\hline Chuck roast & Independent & $\begin{array}{c}\text { Chain } \\
\text { A }\end{array}$ & $\begin{array}{c}\text { Voluntary } \\
\text { Chain } \\
\text { A }\end{array}$ \\
\hline Ground beef & $\begin{array}{c}\text { Chain } \\
\text { A }\end{array}$ & $\begin{array}{c}\text { Voluntary } \\
\text { Chain } \\
\text { B }\end{array}$ & Independent \\
\hline
\end{tabular}

$\begin{array}{lccc}\text { Ground chuck } & \text { Chain } & \begin{array}{c}\text { Voluntary } \\ \text { Chain }\end{array} & \text { Independent } \\ & \text { A } & \text { B } & \text { C }\end{array}$

\begin{tabular}{lccc} 
Ground round & Chain & $\begin{array}{c}\text { Voluntary } \\
\text { Chain } \\
\text { A }\end{array}$ & $\begin{array}{c}\text { Independent } \\
\text { A }\end{array}$ \\
Porterhouse steak & $\begin{array}{c}\text { Voluntary } \\
\text { Chain }\end{array}$ & $\begin{array}{c}\text { Chain } \\
\text { A }\end{array}$ & $\begin{array}{c}\text { Independent } \\
\text { A }\end{array}$ \\
\hdashline & & & continued
\end{tabular}


Table 13 continued.

\begin{tabular}{|c|c|c|c|}
\hline Round steak & $\begin{array}{c}\text { Chain } \\
\text { A }\end{array}$ & $\begin{array}{c}\text { Voluntary } \\
\text { Chain } \\
\text { A }\end{array}$ & Independent \\
\hline Rump roast & $\begin{array}{c}\text { Chain } \\
\text { A }\end{array}$ & $\begin{array}{c}\text { Voluntary } \\
\text { Chain } \\
\text { B }\end{array}$ & $\begin{array}{c}\text { Independent } \\
\text { B }\end{array}$ \\
\hline Sirloin steak & Independent & $\begin{array}{c}\text { Chain } \\
\text { A }\end{array}$ & $\begin{array}{c}\text { Voluntary } \\
\text { Chain } \\
\text { A }\end{array}$ \\
\hline Stew & $\begin{array}{c}\text { Chain } \\
\text { A }\end{array}$ & $\begin{array}{c}\text { Voluntary } \\
\text { Chain } \\
\text { B }\end{array}$ & Independent \\
\hline T-Bone steak & $\begin{array}{c}\text { Voluntary } \\
\text { Chain } \\
\text { A }\end{array}$ & $\begin{array}{c}\text { Chain } \\
\text { A }\end{array}$ & $\begin{array}{c}\text { Independent } \\
\mathrm{A}\end{array}$ \\
\hline
\end{tabular}

*Those means with the same letter are not significantly different.

All means are ranked from highest to lowest.

chain, voluntary chain, and independent stores on beef items than on pork and chicken. It is interesting to note that the independent store tended to have lower prices for a majority of the meats analyzed. Of 20 cuts sold in each store type, the independent had lower average prices for 13 , of which 8 were statistically significantly lower than the prices for either the chain or voluntary chain.

\section{Analysis of National Retail and Wholesale Prices}

This section presents results of regression analyses of the meat price data using six different models. Model I regresses the price of local retail meat cuts of beef and pork with the USDA's weighted average price of retail cuts from the carcass. Model II is used to determine the relationships between local retail prices of selected cuts of beef and pork and the USDA's value of the wholesale quantity equivalent to one pound of retail cuts. Models III and IV were similar to the first two except that the national average retail and wholesale prices were lagged one week. For Models V and VI a stepwise technique was used 
with the independent variables including current retail and wholesale prices, the advertised prices, and the retail and wholesale prices lagged from one to six weeks. The stepwise procedure selects the independent variables which are "best" from explanatory and statistical significance.

\section{Retail Prices}

Model I was a regression model of the form:

$$
R P=a-b_{1} R P_{u s d a}+b_{2} S P
$$

where

a $\quad=$ intercept

$\mathrm{RP} \quad=$ retail price of a meat item in the local area.

$\mathrm{RP}_{\text {usda }}=$ estimated weighted average price of retail cuts from the carcass.

$\mathrm{SP}=$ dummy variable with the value of 1 or 2 , where $1=$ advertised price and $2=$ unadvertised price. In the GLM procedure using SAS the classes statement results in the highest valued classification, in this case 2 , being excluded from the model, i.e., being treated as 0 in the usual 0-1 dummy variable approach to regression.

$b_{1} \quad=$ regression coefficient for the USDA average retail price of the type of meat (beef or pork).

$b_{2} \quad=$ regression coefficient for the dummy variable of the advertised price of a meat item.

Appendix Tables 1 and 2 present the regression results for the pork and beef cuts, respectively. The columns contain stores while the rows contain regression data for each meat cut. The results do indicate, regardless of store or pork cut, that there is not a strong relationship between local retail pork prices and the USDA's weighted average national price of retail pork cuts from the carcass. However, the results do tend to indicate that there are relationships between local beef retail prices and the USDA's weighted average national price of retail beef cuts. The coefficient of determination $\left(R^{2}\right)$ was statistically significant $(\alpha=.01)$ for the equation for each store with a range of values from .48 to .98 . The coefficients of the dummy variables for nearly all the advertised prices were negative, indicating that when a meat was advertised its price was less than the local retail price for that cut. The discount ranged from .48 to $\$ 1.06$ per pound for beef and $\$ .03$ to $\$ 1.40$ for pork. ${ }^{2}$

${ }^{2}$ In some cases, coefficients for the dummy variables were positive although not statistically significantly different from zero. 
Appendix Table 3 contains the results for the beef cuts regardless of store. The prices for all of the beef cuts were significantly related to the USDA's national retail average price $(\alpha=.01)$. The coefficient of determination $\left(\mathrm{R}^{2}\right)$ ranged from .11 for chuck roast and ground round to .71 for round steak. As expected, the advertised price (dummy variable) was negative in all cases. The discount ranged from about 14 cents per pound for ground beef to 72 cents for round steak.

\section{Wholesale Prices}

The regression equations for the wholesale price model were similar to those for the retail model but with average USDA wholesale prices substituted for the USDA retail prices. Appendix Tables 4 and 6 present the results of the regression analyses for the pork and beef cuts, respectively.

Local pork prices appear to be statistically significantly related to the wholesale price. The coefficients of determination, $\mathrm{R}^{2}$, ranged from .56 to .82. In all cases where a statistically significant relationship exists, the coefficients for advertised prices (dummy variable) were less than the average retail price.

The results also indicate that local retail beef prices tend to be related to the USDA's value of wholesale quantity equivalent to one pound of retail cuts. The coefficients for prices of round steak were statistically significantly related for all nine stores. The advertised price for round steak ranged from 48 cents to $\$ 1.04$ per pound less than the unadvertised prices. Many of the other beef cuts also had statistically significant relationships to the wholesale prices in all the stores.

Appendix Table 6 contains the results for all the beef cuts regardless of store. These tend to confirm that there are relationships between the local retail beef prices and USDA's wholesale values. The $\mathrm{R}^{2}$ values range from .10 for chuck roast to .71 for round steak. The advertised price (dummy variable) was negative in all cases. The discount ranged from 14 to 73 cents per pound.

\section{Lagged Price Models}

Model III was similar to the other regression models except that the independent variables were national retail prices lagged one week.

Appendix Table 7 contains the results of the regression analysis for this model. The equation was found to be statistically significant at the $\alpha=.01$ for all seven cuts. The coefficient of determination, $\mathrm{R}^{2}$, ranged from .11 for both chuck roast and ground round to .71 for round 
steak. Thus, there is sufficient statistical evidence to suggest relationships exist between the local retail price of beef cuts and USDA's weighted average price of retail cuts lagged one week. However, the results for this model were not markedly superior to those for the model with current retail prices.

Appendix Table 8 shows the results of the regression analysis for Model IV, where USDA's wholesale price was lagged one week for seven cuts of beef. There again appears to be sufficient evidence to suggest that relationships exist between the local retail price and the USDA's wholesale price lagged one week. The $\mathrm{R}^{2}$ values ranged from .11 for both ground round and chuck roast to .71 for round steak. Again, the lagged price model was not superior in explanatory power or statistical significance to the model with current week wholesale prices.

\section{Stepwise Models}

The national retail prices of beef were lagged up to six weeks and tested using a stepwise regression procedure (Appendix Tables 9 and 10). The stepwise procedure confirms the previous model results. In almost all cases the advertised price (SP) variable came into the equations and was highly statistically significant. The other variables, USDA's lagged retail prices, the lagged variables, frequently entered the equations, but no single length of lag appeared to dominate the results. The lagged variables entered the equations more frequently than current values and the one week lag occurred more often than the other lag lengths. The results tend to indicate that local beef prices are somewhat more related to national retail prices in the preceding weeks than to those of the current week. However, as in the one week lag models, the results were not notably superior.

When USDA's wholesale price was lagged up to six weeks for Model VI, using the stepwise procedure, the results again indicate that the advertised price (SP) is a statistically important variable and also that the lagged wholesale prices frequently are statistically significant (Appendix Tables 11 and 12). The results follow a pattern similar to those of the lagged retail prices, but with a tendency to have a slightly lower overall statistical significance for the lagged variables. Current wholesale prices enter the equation more often than in the retail model and no one length of lag dominated the results. 


\section{Conclusions}

Grocery stores in the local study area seem not to compete strongly on prices of meat although all use advertised specials and price differences do exist. In all cases, the average advertised prices were lower than the unadvertised prices. Gray and Anderson found that advertised prices were approximately 20 percent less, while Rhodes and Abou-Bakr found that advertised prices averaged about 9 percent less than unadvertised prices. This study indicated that the advertised prices were approximately 16 percent less than the unadvertised price. Pork averaged 15 percent less while beef and chicken averaged 16 and 18 percent less, respectively. There were some differences among advertising practices for meat among the stores, although most appeared to be similar. One store tended to have low advertised, but high regular prices, while another tended to practice the opposite. Another store tended to have low advertised prices but to vary in ranking for unadvertised prices. There were some differences between the advertised price means of the same meat items among the other stores, but there were no distinct patterns, although some meat products were treated differently by particular stores.

Beef was advertised more than pork or chicken. This was expected as consumers have demonstrated preferences for beef. The higher priced beef cuts such as rump roasts, porterhouse steak, sirloin steak, and T-Bone steak tend to be discounted more when advertised than the other beef cuts. These items also tend to have a higher price per pound and may have to be discounted more to attract the consumer. Popular items such as round steak and many of the chicken cuts also are discounted relatively more. This is not surprising as these cuts are often bought by the consumer and thus may be used in the stores' pricing policies to attract more customers.

When the stores were grouped by type, it was found that there were no statistically significant differences between the three store types on the mean price of most meat items. This indicates that their pricing strategies are similar although their management and ownership differ.

There were statistically significant differences between the overall price level in each of the stores for some meat items, but few apparent or consistent patterns. However, there was a tendency for one of the voluntary chains to have lower mean unadvertised prices on most items. When this store advertised its meat, its percent discount was not as large as the other stores. 
The lagged and stepwise models used in this study reemphasized the important role that advertised prices play in explaining price variation among beef cuts. The local beef prices also were more related to lagged national retail beef prices than to lagged national wholesale prices, a somewhat surprising result, since changes in wholesale prices should precede retail price adjustments.

The evidence from this study suggests that local pork prices are not as related to either national retail or wholesale prices as local beef prices. Beef prices in this local area tended to be correlated with national average prices, but the lack of really strong, close correlations indicates that local factors may tend to have an important influence on the prices in a particular store in the local area. 


\section{Bibliography}

Bell, M. Marketing Concepts and Strategy, 2nd ed. New York: Houghton Mifflin Co., 1972.

Boone, L. and D. Kurtz. Contemporary Marketing. Hinsdale, Ill.: The Dryden Press, 1974.

Bussel, N. 1974 Progressive Grocer's Marketing Guidebook. New York: Progressive Grocer Co., 1973.

Clodius, R. and W. Mueller. "Market Structure Analysis As an Orientation for Research in Agricultural Economics." Journal of Farm Economics, Vol. XLIII, No. 3, August, 1961.

Conner, J. "Advertising, Promotion and Competition: A Survey with Special Reference to Food." Agricultural Economics Research, Vol. 33, No. 1, January 1981.

Draper, N. and H. Smith. Applied Regression Analysis. New York: John Wiley and Sons, Inc., 1967.

Dunn, S. and A. Barban. Advertising: Its Role in Modern Marketing. Hinsdale, Ill. The Dryden Press, 1974.

Engel, J., H. Wales and M. Warshaw. Promotional Strategy. Homewood, Ill., Richard D. Irwin, Inc., 1971.

Gallo, A. "Food Advertising." National Food Review. Washington: U.S. Government Printing Office, Winter, 1981.

Gaw, W. Advertising Methods and Media.San Francisco: Wadsworth Publishing Co., Inc., 1961.

Gray, R. and R. Anderson. Advertised Specials and Local Competition Among Supermarkets. Stanford University Food Research Institute Studies, Vol. III, May, 1962.

Helwig, J. and K. Council, eds. SAS User's Guide, 1979 edition. Cary, N.C.: SAS Institute, Inc., 1979.

Holdren, B. The Structure of a Retail Market and the Market Behavior of Retail Units. Englewood Cliffs, N.J.: Prentice-Hall, 1961.

Kelley, H. "Cost-Price Squeeze: How to Establish Selling Prices." in Pricing Strategy, edited by B. Taylor and G. Wills. New York: Brandon Systems Press, Inc., 1970.

Kohls, R. and W. Downey. Marketing of Agricultural Products. New York: MacMillan Publishing Co., Inc., 1972. 
Manchester, A. "Food Groups in the New Consumer Price Index." National Food Review, Washington: U.S. Government Printing Office, April, 1978.

Martin, L., ed. A Survey of Agricultural Economics Literature, Vol. I. Minneapolis: University of Minnesota Press, 1977.

Nelson, P. and L. Preston. Price Merchandising in Food Retailing: A Case Study. Institute of Business and Economic Research. Berkeley: University of California, 1966.

Preston, L. "Markups, Leaders and Discrimination in Retail Pricing." Journal of Farm Economics, Vol. XLIV, No. 2. May 1962.

Progressive Grocer. New York: Maclean Hunter Media, Inc., April Annual Report, 1981.

Rhodes, J. and A. Abou-Bakr. "Advertising-Pricing Conduct in Meat Retailing." Southern Journal of Agricultural Economics, Vol. 6, No. 2, December, 1974.

Rhodes, J., R. Schneider, D. Smith, W. Stringer and G. Grimes. Customer Responses to Retail Meat Prices and Ads. Missouri Agricultural Experiment Station Research Bulletin 1006, 1974.

Rhodes, J. The Agricultural Marketing System. Columbus, Ohio: Grid Publishing, Inc., 1978.

Settel, I. Effective Retail Advertising. New York: Fairchild Publications, Inc., 1950.

Steel, R. and J. Torrie. Principles and Procedures of Statistics. New York: McGraw-Hill Book Co., 1960.

U.S. Department of Agriculture. 1980 Handbook of Agricultural Charts. Agriculture Handbook No. 574, Washington: U.S. Government Printing Office, 1980.

U.S. Department of Commerce. 1980 Census of Population and Housing, Preliminary Reports. Bureau of the Census, Washington, D.C., 1980. 
APPENDIX 
Table 1.

Multiple Regression Model Results for National Retail Pork Prices by Store. ${ }^{a}$

\begin{tabular}{|c|c|c|c|c|c|c|c|c|c|c|c|c|}
\hline \multirow[b]{2}{*}{ Meat Item } & \multicolumn{3}{|c|}{$\begin{array}{c}\text { Regional Chain } \\
1\end{array}$} & \multicolumn{3}{|c|}{$\begin{array}{c}\text { Voluntary Chain } \\
2\end{array}$} & \multicolumn{3}{|c|}{$\begin{array}{c}\text { Voluntary Chain } \\
3\end{array}$} & \multicolumn{3}{|c|}{$\begin{array}{c}\text { National Chain } \\
4\end{array}$} \\
\hline & $\mathbf{R}^{2}$ & RP & SP & $\mathbf{R}^{2}$ & RP & $\mathrm{SP}$ & $\mathbf{R}^{2}$ & RP & SP & $\mathbf{R}^{2}$ & RP & SP \\
\hline Picnic & & b & & & & & & & & $.63^{* *}$ & 1.12 & $-14.39 * *$ \\
\hline $\begin{array}{l}\text { Spare ribs } \\
\text { Pork steak }\end{array}$ & .16 & -.30 & -9.19 & .12 & -1.71 & -3.47 & $.78^{* *}$ & .16 & $-15.32^{* *}$ & $.33^{*}$ & .65 & -23.66 \\
\hline
\end{tabular}

\begin{tabular}{|c|c|c|c|c|c|c|c|c|c|c|c|c|c|c|c|}
\hline \multirow[b]{2}{*}{ Meat Item } & \multicolumn{3}{|c|}{$\begin{array}{c}\text { National Chain } \\
5\end{array}$} & \multicolumn{3}{|c|}{$\begin{array}{c}\text { Regional Chain } \\
6\end{array}$} & \multicolumn{3}{|c|}{$\begin{array}{c}\text { Regional Chain } \\
7\end{array}$} & \multicolumn{3}{|c|}{$\begin{array}{c}\text { Independent } \\
8 \\
\end{array}$} & \multicolumn{3}{|c|}{$\begin{array}{c}\text { Voluntary Chain } \\
9 \\
\end{array}$} \\
\hline & $\mathrm{R}^{2}$ & $\mathrm{RP}$ & SP & $\mathrm{R}^{2}$ & $\mathrm{RP}$ & SP & $\mathrm{R}^{2}$ & RP & SP & $\mathbf{R}^{2}$ & RP & $\mathrm{SP}$ & $\mathrm{R}^{2}$ & $\mathrm{RP}$ & SP \\
\hline Picnic & $.89 * *$ & $1.13^{\star *}$ & $-6.08^{*}$ & & b & & & & & & & & & & \\
\hline Spare ribs & $.77^{* *}$ & $1.30^{* *}$ & $-22.11^{* *}$ & $.40 * *$ & $-.38^{* *}$ & -1.69 & .01 & -.19 & -.88 & .09 & -.79 & -2.19 & .02 & .22 & 3.07 \\
\hline Pork steak & .41 & -.06 & -30.12 & .50 & .86 & -33.62 & $.90^{* *}$ & $1.00^{* *}$ & $-40.36^{* *}$ & $.59^{* *}$ & -.41 & $-28.32^{* *}$ & .33 & 1.19 & 9.93 \\
\hline
\end{tabular}

*Significant @ =.05

**Significant @=.01

${ }^{\text {a } T h e ~ i n d e p e n d e n t ~ v a r i a b l e s ~ u s e d ~ w e r e: ~}$

$\mathrm{RP}=$ Estimated weighted a verage price of retail cuts from the carcass for pork.

$\mathrm{SP}=$ Dummy variable with values 1 and 2 .

1 = advertised price of a meat item.

2 = unadvertised price of a meat item.

${ }^{\mathrm{b}}$ Blank space indicates that the meat item was not included in this model. 
Table 2.

Multiple Regression Model Results for National Retail Beef Prices by Store. ${ }^{a}$

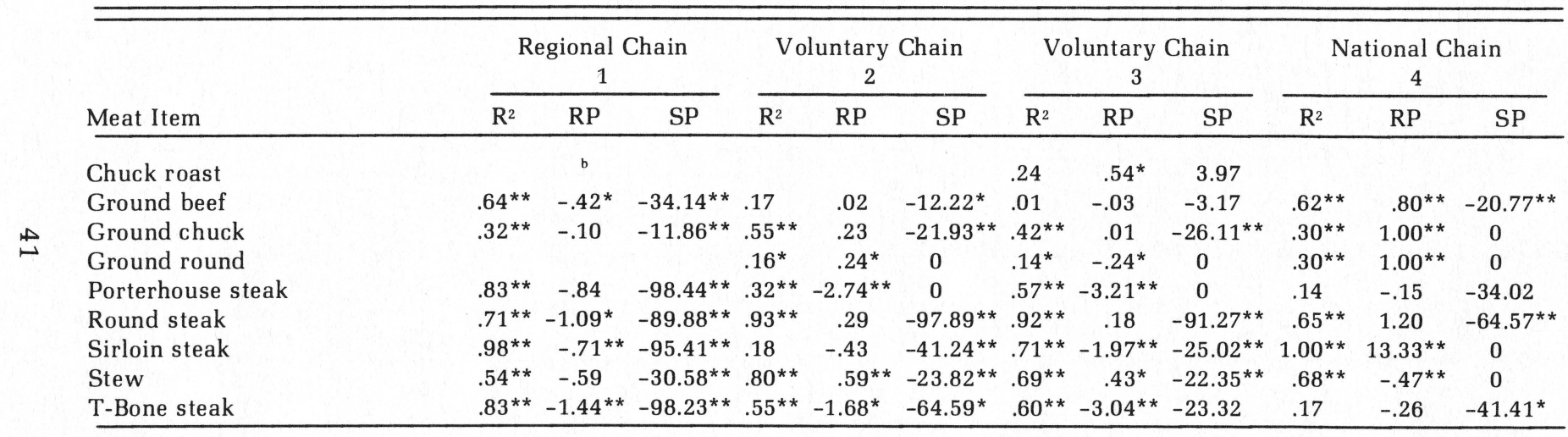


Table 2 Continued.

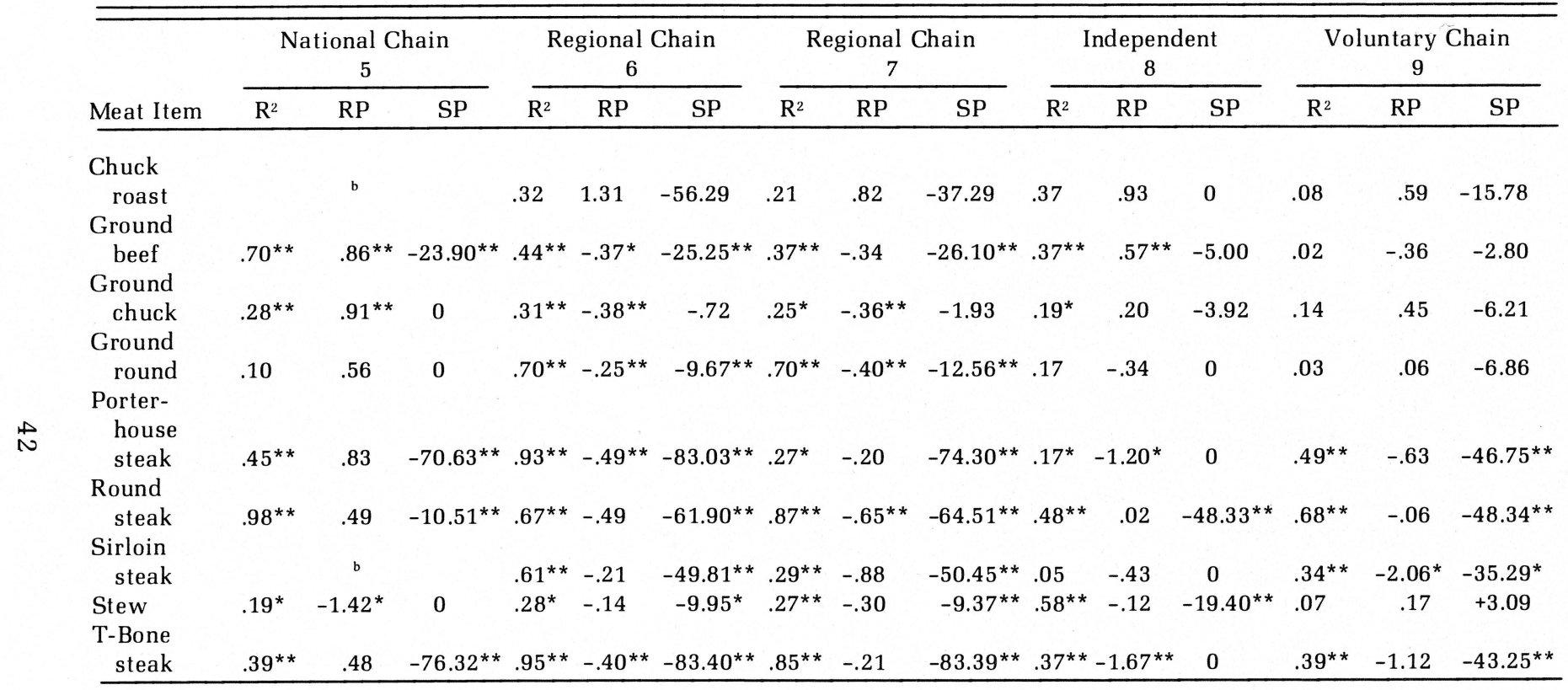

*Significant @=.05

**Significant @=.01

${ }^{\text {a }}$ The independent variables used were:

$\mathrm{RP}=$ Estimated weighted a verage price of retail cuts from the carcass for beef.

$\mathrm{SP}=$ Dummy variable with values 1 and 2 .

1 = advertised price of a meat item.

2 = unadvertised price of a meat item.

${ }^{\mathrm{b}}$ Blank space indicates that the meat item was not included in this model. 
Table 3.

Multiple Regression Model Results for National Retail Beef Prices Regardless of Store. ${ }^{a}$

\begin{tabular}{llll}
\hline \hline Meat Item & $\mathrm{R}^{2}$ & $\mathrm{RP}$ & $\mathrm{SP}$ \\
\hline Chuck roast & $.11^{* *}$ & .72 & $-23.5^{*}$ \\
Ground beef & $.19^{* *}$ & .10 & $-13.66^{* *}$ \\
Ground chuck & $.19^{* *}$ & .18 & $-17.62^{* *}$ \\
Ground round & $.11^{* *}$ & .07 & $-19.41^{* *}$ \\
Porterhouse steak & $.37^{* *}$ & $-.98^{* *}$ & $-59.74^{* *}$ \\
Round steak & $.71^{* *}$ & -.11 & $-72.38^{* *}$ \\
Sirloin steak & $.40^{* *}$ & $-.87^{* *}$ & $-53.73^{* *}$ \\
Stew & $.24^{* *}$ & -.15 & $-27.88^{* *}$ \\
T-Bone steak & $.39^{* *}$ & $-.95^{* *}$ & $-64.00^{* *}$ \\
\hline
\end{tabular}

*Significant @=.05

**Significant @=.01

${ }^{a}$ The independent variables used were:

$\mathrm{RP}=$ Estimated weighted a verage price of retail cuts from the carcass for beef.

$\mathrm{SP}=$ Dummy variable with values 1 and 2.

1 = advertised price of a meat item.

2 = unadvertised price of a meat item. 
Table 4.

Multiple Regression Model Results for National Wholesale Pork Prices by Store. ${ }^{a}$

\begin{tabular}{|c|c|c|c|c|c|c|c|c|c|c|c|c|}
\hline \multirow[b]{2}{*}{ Meat item } & \multicolumn{3}{|c|}{$\begin{array}{c}\text { Regional Chain } \\
1 \\
\end{array}$} & \multicolumn{3}{|c|}{$\begin{array}{c}\text { Voluntary Chain } \\
2\end{array}$} & \multicolumn{3}{|c|}{$\begin{array}{c}\text { Voluntary Chain } \\
3\end{array}$} & \multicolumn{3}{|c|}{$\begin{array}{c}\text { National Chain } \\
4\end{array}$} \\
\hline & $\mathrm{R}^{2}$ & WP & SP & $\mathrm{R}^{2}$ & WP & SP & $\mathrm{R}^{2}$ & WP & SP & $\mathbf{R}^{2}$ & WP & $\mathrm{SP}$ \\
\hline Picnic & & b & & & & & & & & $.56^{* *}$ & $1.15^{* *}$ & $-15.83^{* *}$ \\
\hline $\begin{array}{l}\text { Spare ribs } \\
\text { Pork steak }\end{array}$ & .14 & -.13 & -9.77 & .04 & -.28 & -10.56 & $.77^{* *}$ & .17 & $-15.18^{* *}$ & .28 & .45 & -27.23 \\
\hline
\end{tabular}

Table 4 Continued.

\begin{tabular}{|c|c|c|c|c|c|c|c|c|c|c|c|c|c|c|c|}
\hline \multirow[b]{2}{*}{ Meat Item } & \multicolumn{3}{|c|}{$\begin{array}{c}\text { National Chain } \\
5 \\
\end{array}$} & \multicolumn{3}{|c|}{$\begin{array}{c}\text { Regional Chain } \\
6 \\
\end{array}$} & \multicolumn{3}{|c|}{$\begin{array}{c}\text { Regional Chain } \\
7 \\
\end{array}$} & \multicolumn{3}{|c|}{$\begin{array}{c}\text { Independent } \\
8 \\
\end{array}$} & \multicolumn{3}{|c|}{$\begin{array}{c}\text { Voluntary Chain } \\
9 \\
\end{array}$} \\
\hline & $\mathrm{R}^{2}$ & WP & SP & $\mathrm{R}^{2}$ & WP & SP & $\mathrm{R}^{2}$ & WP & SP & $\mathrm{R}^{2}$ & WP & SP & $\mathrm{R}^{2}$ & WP & SP \\
\hline Picnic & $.82^{* *}$ & $1.32^{\star *}$ & $-7.49^{*}$ & & & & & & & & & & & & \\
\hline Spare ribs & $.77^{* *}$ & $1.74^{\star *}$ & $-22.72^{\star *}$ & $.37^{* *}$ & $-.44^{* *}$ & -3.33 & .02 & .44 & -1.40 & .03 & -.30 & 7.05 & .02 & .21 & 2.95 \\
\hline Pork steak & .50 & 1.41 & -27.88 & $.49^{* *}$ & 1.03 & $-31.91 * *$ & $.81^{* *}$ & $.76^{*}$ & $-38.24^{* *}$ & $.57^{* *}$ & -.39 & $-28.89^{* *}$ & .23 & .94 & 15.82 \\
\hline
\end{tabular}

*Significant @ =.05

**Significant @ =.01

${ }^{\mathrm{a}}$ The independent variables used were:

WP = Estimated value of wholesale quantity equivalent to one pound of retail cuts.

$\mathrm{SP}=$ Dummy variable with values 1 and 2 .

1 = advertised price of a meat item.

2 = unadvertised price of a meat item.

${ }^{\mathrm{h}}$ Blank space indicates that the meat item was not included in this model. 
Table 5.

Multiple Regression Model Results for National Wholesale Beef Prices by Store. ${ }^{a}$

\begin{tabular}{|c|c|c|c|c|c|c|c|c|c|c|c|c|}
\hline \multirow[b]{2}{*}{ Meat Item } & \multicolumn{3}{|c|}{$\begin{array}{c}\text { Regional Chain } \\
1 \\
\end{array}$} & \multicolumn{3}{|c|}{$\begin{array}{c}\text { Voluntary Chain } \\
2 \\
\end{array}$} & \multicolumn{3}{|c|}{$\begin{array}{c}\text { Voluntary Chain } \\
3\end{array}$} & \multicolumn{3}{|c|}{$\begin{array}{c}\text { National Chain } \\
4 \\
\end{array}$} \\
\hline & $\mathrm{R}^{2}$ & WP & SP & $\mathrm{R}^{2}$ & WP & SP & $\mathrm{R}^{2}$ & WP & SP & $\mathrm{R}^{2}$ & WP & $\mathrm{SP}$ \\
\hline Chuck roast & & b & & & & & .15 & .52 & 3.51 & & & \\
\hline Ground beef & $.76^{* *}$ & $-.84^{* *}$ & $-.37 .64 * *$ & $.21 *$ & -.27 & $-12.91^{*}$ & .05 & -.25 & -3.73 & $.54^{* *}$ & $.73^{*}$ & $-19.68^{* *}$ \\
\hline Ground chuck & $.42^{* *}$ & -.38 & $-10.26^{* *}$ & $.52^{* *}$ & .10 & $-21.35^{* *}$ & $.42^{* *}$ & -.01 & $-26.03^{* *}$ & .12 & .80 & 0 \\
\hline Ground round & & & & .06 & .18 & 0 & $.13^{*}$ & $-.28^{\star}$ & 0 & .06 & .58 & 0 \\
\hline Porterhouse steak & $.84^{* *}$ & $-1.30^{*}$ & $-97.57^{* *}$ & $.30 * *$ & $-3.26^{* *}$ & 0 & $.45^{* *}$ & $-3.57^{* *}$ & 0 & .15 & -.71 & -32.56 \\
\hline Round steak & $.68^{* *}$ & $-1.04^{*}$ & $-88.01^{* *}$ & $.93^{* *}$ & -.14 & $-97.21^{* *}$ & $.92^{* *}$ & .01 & $-91.04^{* *}$ & $.66^{\star *}$ & 1.98 & $-60.37^{* *}$ \\
\hline Sirloin steak & $.98^{* *}$ & $-.82^{* *}$ & $-94.29^{\star *}$ & $.23^{*}$ & -.99 & $-40.40^{\star}$ & $.74^{* *}$ & $-2.61^{* *}$ & $-24.18^{* *}$ & $1.00^{\star *}$ & $-80.00^{* *}$ & 0 \\
\hline Stew & $.62^{* *}$ & $-1.12^{* *}$ & $-31.36^{\star \star}$ & $.75^{\star *}$ & .48 & $-24.53^{* *}$ & $.66^{* *}$ & .31 & $-23.13^{\star *}$ & $.73^{* *}$ & $-.60^{\star *}$ & 0 \\
\hline T-Bone steak & $.81^{* *}$ & $-1.49^{* *}$ & $-94.50^{* *}$ & $.55^{* *}$ & $-2.11^{\star}$ & $-63.08^{\star}$ & $.45^{* *}$ & $-3.13^{\star *}$ & -29.04 & .17 & -.26 & $-40.76^{*}$ \\
\hline
\end{tabular}


Table 5 Continued.

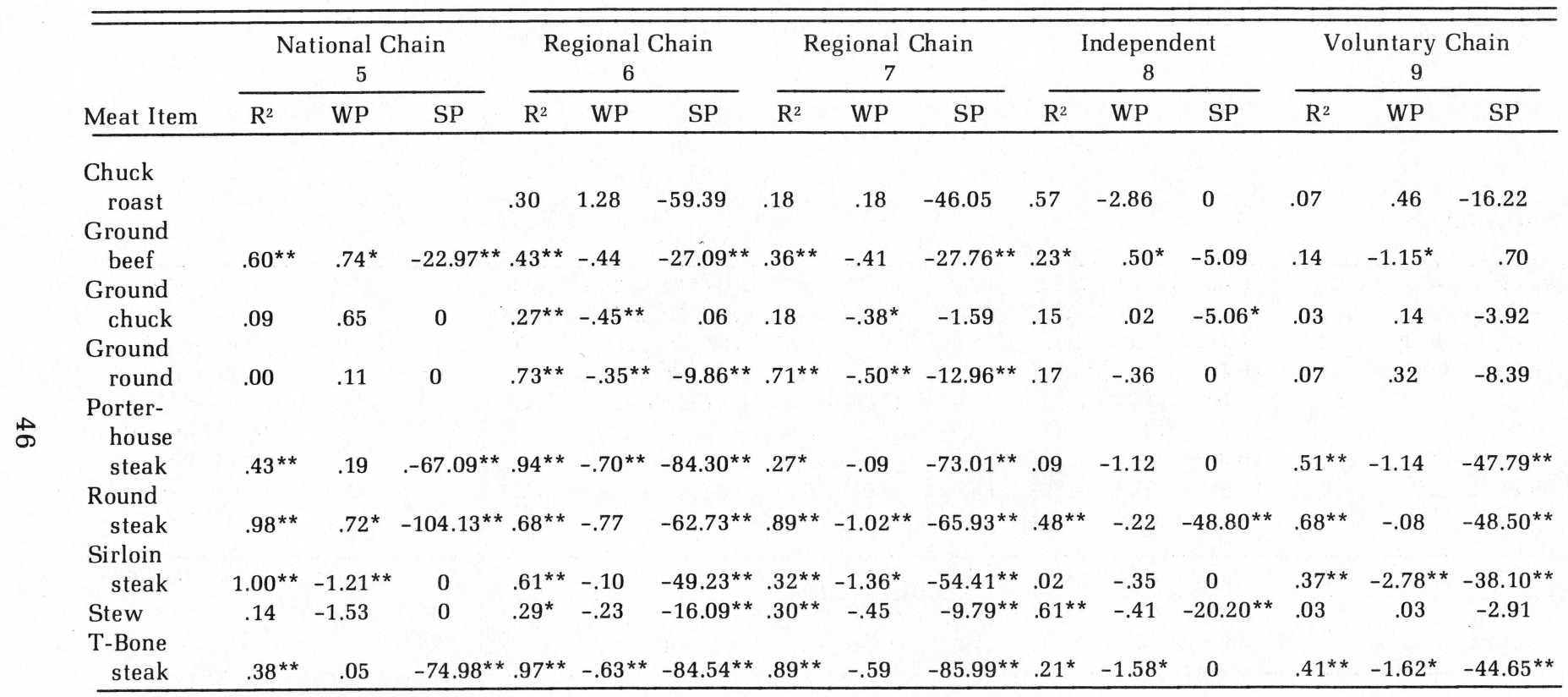

*Significant @ =.05

**Significant @=.01

${ }^{a}$ The independent variables used were:

$\mathrm{WP}=$ Estimated value of wholesale quantity equivalent to one pound of retail cuts.

$\mathrm{SP}=$ Dummy variable with values 1 and 2.

1 = advertised price of a meat item.

2 = unadvertised price of a meat item.

${ }^{b}$ Blank space indicates that the meat item was not included in this model. 
Table 6.

Multiple Regression Model Results for National Wholesale Beef Prices Regardless of Store. ${ }^{\mathrm{a}}$

\begin{tabular}{llcc}
\hline Meat Item & $\mathrm{R}^{2}$ & $\mathrm{WP}$ & $\mathrm{SP}$ \\
\hline Chuck roast & $.10^{*}$ & .64 & $-23.91^{*}$ \\
Ground beef & $.19^{* *}$ & -.10 & $-13.70^{* *}$ \\
Ground chuck & $.19^{* *}$ & .02 & $-17.55^{* *}$ \\
Ground round & $.11^{* *}$ & -.14 & $-18.80^{* *}$ \\
Porterhouse steak & $.38^{* *}$ & $-1.29^{* *}$ & $-60.21^{* *}$ \\
Round steak & $.71^{* *}$ & -.25 & $-72.62^{* *}$ \\
Sirloin steak & $.42^{* *}$ & $-1.23^{* *}$ & $-54.32^{* *}$ \\
Stew & $.24^{* *}$ & -.35 & $-28.11^{* *}$ \\
T-Bone steak & $.39^{* *}$ & $-1.21^{* *}$ & $-62.48^{* *}$ \\
\hline
\end{tabular}

*Significant @=.05

**Significant @ =.01

${ }^{a}$ The independent variables used were:

$W P=$ Estimated value of wholesale quantity equivalent to one pound of retail cuts.

$\mathrm{SP}=$ Dummy variable with values 1 and 2.

$1=$ advertised price of a meat item.

2 = unadvertised price of a meat item.

\section{Table 7.}

Multiple Regression Model Results Lagged for National Retail Beef Prices Regardless of Store. ${ }^{a}$

\begin{tabular}{llll}
\hline \hline Meat Item & $\mathrm{R}^{2}$ & $\mathrm{RPL}_{1}$ & $\mathrm{SP}$ \\
\hline Chuck roast & $.11^{* *}$ & .69 & $-23.94^{*}$ \\
Ground beef & $.19^{* *}$ & .11 & $-13.62^{* *}$ \\
Ground chuck & $.19^{* *}$ & .17 & $-17.63^{* *}$ \\
Ground round & $.11^{* *}$ & .09 & $-19.45^{* *}$ \\
Porterhouse steak & $.36^{* *}$ & $-.82^{* *}$ & $-59.30^{* *}$ \\
Round steak & $.71^{* *}$ & -.09 & $-72.39^{* *}$ \\
Sirloin steak & $.39^{* *}$ & $-.78^{* *}$ & $-53.65^{* *}$ \\
\hline
\end{tabular}

*Significant @=.05

**Significant @=.01

${ }^{a}$ The independent variables used were:

$\mathrm{RPL}_{1}=$ Estimated weighted average of retail cuts from the carcass lagged one week.

$\mathrm{SP}=$ Dummy variable with values 1 and 2 .

$1=$ advertised price of a meat item.

2 = unadvertised price of a meat item. 
Table 8.

Multiple Regression Model Results Lagged for National Wholesale Beef Prices Regardless of Store.

\begin{tabular}{llcc}
\hline \hline Meat Item & $\mathrm{R}^{2}$ & $\mathrm{WPL}_{1}$ & $\mathrm{SP}$ \\
\hline Chuick roast & $.11^{*}$ & .71 & $-23.74^{*}$ \\
Ground beef & $.18^{* *}$ & -.04 & $-13.65^{* *}$ \\
Ground chuck & $.19^{* *}$ & .09 & $-17.56^{* *}$ \\
Ground round & $.11^{* *}$ & -.05 & $-19.02^{* *}$ \\
Porterhouse steak & $.37^{* *}$ & $-1.18^{* *}$ & $-60.57^{* *}$ \\
Round steak & $.71^{* *}$ & -.34 & $-72.80^{* *}$ \\
Sirloin steak & $.43^{* *}$ & $-1.24^{* *}$ & $-54.70^{* *}$ \\
\hline
\end{tabular}

*Significant @ =.05

**Significant @ =.01

The independent variables used were:

$W P L_{1}=$ Estimated value of wholesale quantity equivalent to one pound of retail cuts lagged one week.

$\mathrm{SP}=$ Dummy variable with values 1 and 2.

1 = advertised price of a meat item.

2 = unadvertised price of a meat item. 


\section{Table 9.}

Statistical Analysis From the General Linear Models Procedure for Independent Variables Selected by Stepwise Procedures for Factors Affecting the Dependent Variable: Local Retail Prices of a Meat Item. ${ }^{a}$

\begin{tabular}{|c|c|c|c|c|c|c|}
\hline \multirow{2}{*}{$\frac{\text { Meat Item }}{\text { Ground beef }}$} & \multicolumn{2}{|c|}{$\begin{array}{l}\text { Regional } \\
\text { Chain } \\
\text { Store } 1 \\
\end{array}$} & \multicolumn{2}{|c|}{$\begin{array}{c}\text { Voluntary } \\
\text { Chain } \\
\text { Store } 2 \\
\end{array}$} & \multicolumn{2}{|c|}{$\begin{array}{c}\text { National } \\
\text { Chain } \\
\text { Store } 4 \\
\end{array}$} \\
\hline & $\mathrm{R}^{2}$ & $.65^{* *}$ & $\mathrm{R}^{2}$ & $.17^{*}$ & $\mathrm{R}^{2}$ & $.67^{* *}$ \\
\hline & $\mathrm{SP}$ & $33.61^{* *}$ & SP & $12.24^{*}$ & SP & $18.39 * *$ \\
\hline & $\mathrm{RPL}_{1}$ & $-.42 * *$ & & & $\mathrm{RPL}_{2}$ & $1.77^{* *}$ \\
\hline & & & & & $\mathrm{RPL}_{4}$ & -1.01 \\
\hline \multirow[t]{2}{*}{ Ground chuck } & $\mathrm{R}^{2}$ & $.30 * *$ & $\mathrm{R}^{2}$ & $.56 * *$ & $\mathrm{R}^{2}$ & $.30 * *$ \\
\hline & SP & 12.17 ** & $\begin{array}{l}\mathrm{SP} \\
\mathrm{RPL}_{4}\end{array}$ & $\begin{array}{c}22.91 * * \\
.28\end{array}$ & $\mathrm{RP}$ & $1.01^{* *}$ \\
\hline \multirow[t]{3}{*}{ Ground round } & b & & $\mathrm{R}^{2}$ & $.16^{*}$ & $\mathrm{R}^{2}$ & $.52^{* *}$ \\
\hline & & & $\mathrm{RP}$ & $.24^{*}$ & $\mathrm{RPL}_{1}$ & $3.61^{* *}$ \\
\hline & & & & & $\mathrm{RPL}_{3}$ & $-2.67^{*}$ \\
\hline \multirow{4}{*}{$\begin{array}{l}\text { Porterhouse } \\
\text { steak }\end{array}$} & $\mathrm{R}^{2}$ & $.83^{* *}$ & $\mathrm{R}^{2}$ & $.60 * *$ & $\mathrm{R}^{2}$ & .14 \\
\hline & $\mathrm{RP}$ & -.84 & $\mathrm{RP}$ & $-4.78^{*}$ & SP & 34.69 \\
\hline & SP & $98.44^{* *}$ & $\mathrm{RPL}_{2}$ & $9.08^{* *}$ & & \\
\hline & & & $\mathrm{RPL}_{3}$ & $-7.14^{* *}$ & & \\
\hline \multirow[t]{3}{*}{ Round steak } & $\mathrm{R}^{2}$ & $.72^{* *}$ & $\mathrm{R}^{2}$ & $.93^{* *}$ & $\mathrm{R}^{2}$ & $.68^{* *}$ \\
\hline & $\mathrm{SP}$ & $88.46^{* *}$ & SP & $97.40^{* *}$ & SP & $65.25^{* *}$ \\
\hline & $\mathrm{RPL}_{1}$ & $-1.09 * *$ & & & $\mathrm{RPL}_{1}$ & 1.51 \\
\hline \multirow[t]{3}{*}{ Sirloin steak } & $\mathrm{R}^{2}$ & $.99 * *$ & $\mathrm{R}^{2}$ & $.16^{*}$ & $\mathrm{R}^{2}$ & $1.00 * *$ \\
\hline & SP & 96.50 ** & SP & $41.54^{*}$ & $\mathrm{RP}$ & $13.33^{* *}$ \\
\hline & $\mathrm{RPL}_{2}$ & $-.88^{* *}$ & & & & \\
\hline \multirow[t]{3}{*}{ Stew } & $\mathrm{R}^{2}$ & $.56^{* *}$ & $\mathrm{R}^{2}$ & $.80^{* *}$ & $\mathrm{R}^{2}$ & $.73^{* *}$ \\
\hline & SP & $30.65^{* *}$ & RP & $.59 * *$ & $\mathrm{RPL}_{1}$ & $-.46^{* *}$ \\
\hline & $\mathrm{RPL}_{1}$ & $-.69 *$ & SP & $23.82^{* *}$ & & \\
\hline \multirow[t]{3}{*}{ T-Bone steak } & $\mathrm{R}^{2}$ & $.83^{* *}$ & $\mathrm{R}^{2}$ & $.57 * *$ & $\mathrm{R}^{2}$ & $.17^{*}$ \\
\hline & SP & $97.89 * *$ & SP & $73.39 * *$ & SP & $42.19^{*}$ \\
\hline & $\mathrm{RPL}_{3}$ & $-1.49 * *$ & $\mathrm{RPL}_{5}$ & $-1.61^{*}$ & & \\
\hline
\end{tabular}

\footnotetext{
*Significant @=.05
}

**Significant @ =.01

${ }^{\text {a }}$ The independent variables used were:

$R_{P L}-R_{1} L_{6}=$ Estimated weighted average price of retail cuts from the carcass lagged from one to six weeks.

${ }^{b}$ Blank space indicates that the meat item was not included in this model. 
Table 10.

Statistical Analysis From the General Linear Models Procedure for Independent Variables Selected by Stepwise Procedures for Factors Affecting the Dependent Variable: Local Retail Prices of a Meat Item. ${ }^{a}$

\begin{tabular}{|c|c|c|c|c|c|c|}
\hline \multirow{2}{*}{$\frac{\text { Meat Item }}{\text { Ground beef }}$} & \multicolumn{2}{|c|}{$\begin{array}{c}\text { Regional } \\
\text { Chain } \\
\text { Store } 1 \\
\end{array}$} & \multicolumn{2}{|c|}{$\begin{array}{c}\text { Voluntary } \\
\text { Chain } \\
\text { Store } 2 \\
\end{array}$} & \multicolumn{2}{|c|}{$\begin{array}{c}\text { National } \\
\text { Chain } \\
\text { Store } 4\end{array}$} \\
\hline & $\mathrm{R}^{2}$ & $.76^{* *}$ & $\mathrm{R}^{2}$ & $.17^{*}$ & $\mathrm{R}^{2}$ & $.71^{* *}$ \\
\hline & WP & $-.84^{* *}$ & SP & $12.23^{*}$ & SP & $19.59^{* *}$ \\
\hline & SP & 37.64 ** & & & $W \mathrm{PL}_{1}$ & -.90 \\
\hline & & & & & $\mathrm{WPL}_{4}$ & $2.89 * *$ \\
\hline & & & & & $\mathrm{WPL}_{5}$ & -1.46 \\
\hline \multirow[t]{4}{*}{ Ground chuck } & $\mathrm{R}^{2}$ & $.54^{* *}$ & $\mathrm{R}^{2}$ & $.51^{* *}$ & $\mathrm{R}^{2}$ & $.25^{* *}$ \\
\hline & WP & $-.97 * *$ & SP & 21.00 ** & $\mathrm{WPL}_{2}$ & $.98^{* *}$ \\
\hline & SP & $11.36^{* *}$ & & & & \\
\hline & $\mathrm{WPL}_{5}$ & $.54^{*}$ & & & & \\
\hline \multirow[t]{3}{*}{ Ground round } & & & $\mathrm{R}^{2}$ & .06 & $\mathrm{R}^{2}$ & $.38^{* *}$ \\
\hline & & & WP & .18 & WP & -1.44 \\
\hline & & & & & $\mathrm{WPL}_{3}$ & $1.87^{* *}$ \\
\hline \multirow[t]{4}{*}{ Round steak } & $\mathrm{R}^{2}$ & $.75^{* *}$ & $\mathrm{R}^{2}$ & $.93^{* *}$ & $\mathrm{R}^{2}$ & $.70^{* *}$ \\
\hline & WP & 3.28 & SP & $97.40 * *$ & SP & $64.71^{* *}$ \\
\hline & SP & 104.01 ** & & & $\mathrm{WPL}_{2}$ & $2.27^{*}$ \\
\hline & $\mathrm{WPL}_{1}$ & $-4.44^{*}$ & & & & \\
\hline \multirow[t]{3}{*}{ Sirloin steak } & $\mathrm{R}^{2}$ & $.99^{* *}$ & $\mathrm{R}^{2}$ & $.16^{*}$ & $\mathrm{R}^{2}$ & 1.00 ** \\
\hline & SP & $94.33^{* *}$ & SP & $41.54^{*}$ & WP & $-80.00 * *$ \\
\hline & WPLs & $-.72 * *$ & & & & \\
\hline \multirow[t]{4}{*}{ Stew } & $\mathrm{R}^{2}$ & $.62 * *$ & $\mathrm{R}^{2}$ & $.85^{* *}$ & $\mathrm{R}^{2}$ & $.78^{* *}$ \\
\hline & WP & $-1.12^{* *}$ & WP & $-1.89 * *$ & WPL $_{6}$ & $-.43^{* *}$ \\
\hline & SP & 31.36 ** & $\mathrm{SP}$ & $23.36^{* *}$ & & \\
\hline & & & $\mathrm{WPL}_{1}$ & $2.37^{* *}$ & & \\
\hline \multirow[t]{3}{*}{ T-Bone steak } & $\mathrm{R}^{2}$ & $.82 * *$ & $\mathrm{R}^{2}$ & $.56^{* *}$ & $\mathrm{R}^{2}$ & $.17^{*}$ \\
\hline & SP & $95.10 * *$ & $\mathrm{SP}$ & $60.53^{*}$ & SP & $42.19^{*}$ \\
\hline & $\mathrm{WPL}_{5}$ & $-1.23^{*}$ & $\mathrm{WPL}_{5}$ & $-1.65^{*}$ & & \\
\hline \multirow{5}{*}{$\begin{array}{l}\text { Porterhouse } \\
\text { steak }\end{array}$} & & & & & & \\
\hline & $\mathrm{R}^{2}$ & $.84 * *$ & $\mathrm{R}^{2}$ & $.60 * *$ & $\mathrm{R}^{2}$ & .14 \\
\hline & WP & $-1.30^{*}$ & $\mathrm{WPL}_{2}$ & -7.80 & $\mathrm{SP}$ & 34.69 \\
\hline & $\mathrm{SP}$ & $97.57^{* *}$ & $\mathrm{WPL}_{3}$ & $12.67^{* *}$ & & \\
\hline & & & WPLs & $-7.33^{* *}$ & & \\
\hline
\end{tabular}

*Significant @ $=.05$

**Significant @=.01

${ }^{a}$ The independent variables used were:

$\mathrm{WPL}_{1}-\mathrm{WPL}_{6}=$ Estimated value of wholesale quantity equivalent to one pound of retail cuts lagged from one to six weeks.

${ }^{\mathrm{b}}$ Blank space indicates that the meat item was not included in this model. 
Table 11.

Stepwise Procedure Model Results for National Retail Beef Prices by Store.

\begin{tabular}{|c|c|c|c|c|c|c|c|c|c|c|}
\hline$\frac{\text { Meat Item }}{\text { Ground beef }}$ & \multicolumn{2}{|c|}{$\begin{array}{c}\text { Voluntary Chain } \\
3 \\
\end{array}$} & \multicolumn{2}{|c|}{ National Chain } & \multicolumn{2}{|c|}{$\begin{array}{c}\text { Regional Chain } \\
6\end{array}$} & \multicolumn{2}{|c|}{$\begin{array}{c}\text { Regional Chain } \\
7\end{array}$} & \multicolumn{2}{|c|}{$\begin{array}{c}\text { Independent } \\
8 \\
\end{array}$} \\
\hline Ground beef & a & & $\begin{array}{l}\mathrm{R}^{2} \\
\mathrm{SP} \\
\mathrm{RPL}_{2}\end{array}$ & $\begin{array}{r}.72 * * \\
23.15^{* *} \\
.89 * *\end{array}$ & $\begin{array}{l}\mathrm{R}^{2} \\
\mathrm{SP} \\
\mathrm{RPL}_{5}\end{array}$ & $\begin{array}{r}.48^{* *} \\
25.66^{* *} \\
-.43^{*}\end{array}$ & $\begin{array}{l}\mathrm{R}^{2} \\
\mathrm{SP} \\
\mathrm{RPL}_{1} \\
\mathrm{RPL}_{5}\end{array}$ & $\begin{array}{r}.47^{* *} \\
27.73^{* *} \\
.86 \\
-1.22^{*}\end{array}$ & $\begin{array}{l}\mathrm{R}^{2} \\
\mathrm{RPL}\end{array}$ & $\begin{array}{l}.36 * * \\
.68^{* *}\end{array}$ \\
\hline Ground chuck & $\begin{array}{l}\mathrm{R}^{2} \\
\mathrm{SP}\end{array}$ & $\begin{array}{r}.42^{* *} \\
26.07^{* *}\end{array}$ & $\begin{array}{l}\mathrm{R}^{2} \\
\mathrm{RPL}_{1}\end{array}$ & $\begin{array}{l}.28^{* *} \\
.92^{* *}\end{array}$ & $\begin{array}{l}\mathrm{R}^{2} \\
\mathrm{RP}\end{array}$ & $\begin{array}{r}.31 * * \\
-.39 * *\end{array}$ & $\begin{array}{l}\mathrm{R}^{2} \\
\mathrm{RPL}_{5}\end{array}$ & $\begin{array}{r}.26^{* *} \\
-.36^{* *}\end{array}$ & $\begin{array}{l}\mathrm{R}^{2} \\
\mathrm{RPL}_{6}\end{array}$ & $\begin{array}{l}.27 * * \\
.45^{* *}\end{array}$ \\
\hline Ground round & $\begin{array}{l}\mathrm{R}^{2} \\
\mathrm{RPL}_{6}\end{array}$ & $\begin{array}{r}.20^{*} \\
-.30^{*}\end{array}$ & $\begin{array}{l}\mathrm{R}^{2} \\
\mathrm{RPL}_{6}\end{array}$ & $\begin{array}{l}.13 \\
.67\end{array}$ & $\begin{array}{l}\mathrm{R}^{2} \\
\mathrm{SP} \\
\mathrm{RPL}_{1} \\
\mathrm{RPL}_{4}\end{array}$ & $\begin{array}{r}.76^{* *} \\
10.60^{* *} \\
-.61^{* *} \\
.38^{*}\end{array}$ & $\begin{array}{l}\mathrm{R}^{2} \\
\mathrm{SP} \\
\mathrm{RPL}_{1} \\
\mathrm{RPL}_{4}\end{array}$ & $\begin{array}{r}.78^{* *} \\
12.64^{* *} \\
-1.06^{* *} \\
.71^{* *}\end{array}$ & & \\
\hline $\begin{array}{l}\text { Porterhouse } \\
\text { steak }\end{array}$ & $\begin{array}{l}\mathrm{R}^{2} \\
\mathrm{RPL}_{3}\end{array}$ & $\begin{array}{r}.63^{* *} \\
-3.30^{* *}\end{array}$ & $\begin{array}{l}\mathrm{R}^{2} \\
\mathrm{SP} \\
\mathrm{RPL}_{6}\end{array}$ & $\begin{array}{r}.98^{* *} \\
105.85^{* *} \\
.56\end{array}$ & $\begin{array}{l}\mathrm{R}^{2} \\
\mathrm{RP} \\
\mathrm{SP} \\
\mathrm{RPL}_{6} \\
\mathrm{R}^{2} \\
\mathrm{SP}\end{array}$ & $\begin{array}{r}.95^{* *} \\
-1.02^{* *} \\
81.21^{* *} \\
.63^{*} \\
.66^{* *} \\
59.53^{* *}\end{array}$ & $\begin{array}{l}\mathrm{R}^{2} \\
\mathrm{SP} \\
\mathrm{RPL}_{1} \\
\mathrm{RPL}_{2}\end{array}$ & $\begin{array}{r}.89^{* *} \\
67.07^{* *} \\
-2.50^{* *} \\
1.96^{*}\end{array}$ & & \\
\hline
\end{tabular}


Table 11 Continued.

\begin{tabular}{|c|c|c|c|c|c|c|c|c|c|}
\hline Meat Item & Volun & $\begin{array}{c}\text { tary Chain } \\
3 \\
\end{array}$ & n Natio & $\begin{array}{l}\text { hal Chain } \\
5\end{array}$ & Regic & $\begin{array}{l}\text { nal Chain } \\
6\end{array}$ & Regi & $\begin{array}{l}\text { nal Chain } \\
7\end{array}$ & $\begin{array}{c}\text { Independent } \\
8 \\
\end{array}$ \\
\hline \multirow[t]{5}{*}{ Sirloin steak } & $\mathrm{R}^{2}$ & $.75^{* *}$ & & & $\mathrm{R}^{2}$ & $.60^{* *}$ & $\mathrm{R}^{2}$ & $.59 * *$ & \\
\hline & SP & $31.11^{* *}$ & & & SP & $48.50 * *$ & SP & $65.43^{* *}$ & \\
\hline & $\mathrm{RPL}_{4}$ & $-1.98 * *$ & & & & & $\mathrm{RPL}_{1}$ & $-6.20 * *$ & \\
\hline & & & & & & & $\mathrm{RPL}_{2}$ & $7.96^{* *}$ & \\
\hline & & & & & & & $\mathrm{RPL}_{4}$ & -2.75 & \\
\hline \multirow[t]{4}{*}{ Stew } & $\mathbf{R}^{2}$ & $.75^{\star *}$ & $\mathbf{R}^{2}$ & $.56^{* *}$ & $\mathrm{R}^{2}$ & $.27^{* *}$ & $\mathrm{R}^{2}$ & $.39 * *$ & \\
\hline & SP & $23.21 * *$ & $\mathrm{RPL}_{5}$ & $-6.75^{* *}$ & SP & $10.71^{* *}$ & $\mathrm{SP}$ & $9.25^{* *}$ & \\
\hline & $\mathrm{RPL}_{2}$ & $-1.40^{* *}$ & $\mathrm{RPL}_{6}$ & $5.82^{* *}$ & & & $\mathrm{RPL}_{3}$ & $-1.34^{*}$ & \\
\hline & $\mathrm{RPL}_{5}$ & $-1.03^{*}$ & & & & & $\mathrm{RPL}_{6}$ & $1.07^{*}$ & \\
\hline \multirow[t]{3}{*}{ T-Bone steak } & $\mathrm{R}^{2}$ & $.63^{\star *}$ & $\mathrm{R}^{2}$ & $.38^{* *}$ & $\mathrm{R}^{2}$ & $.96^{* *}$ & $\mathrm{R}^{2}$ & $.84^{* *}$ & \\
\hline & $\mathrm{RPL}_{2}$ & $-3.21^{* *}$ & $\mathrm{SP}$ & $74.68^{* *}$ & SP & $81.81^{* *}$ & SP & $81.38^{* *}$ & \\
\hline & & & & & $\mathrm{RPL}_{1}$ & $\begin{array}{c}-.82^{* *} \\
53^{*}\end{array}$ & & & \\
\hline
\end{tabular}

*Significant @ =.05

**Significant @ = .01

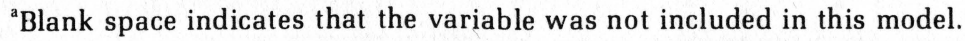


Table 12.

Stepwise Procedure Model Results for National Wholesale Beef Prices by Store.

\begin{tabular}{|c|c|c|c|c|c|c|c|c|c|c|}
\hline$\frac{\text { Meat Item }}{\text { Ground beef }}$ & \multicolumn{2}{|c|}{$\begin{array}{c}\text { Voluntary Chain } \\
3 \\
\end{array}$} & \multicolumn{2}{|c|}{$\begin{array}{c}\text { National Chain } \\
5 \\
\end{array}$} & \multicolumn{2}{|c|}{$\begin{array}{c}\text { Regional Chain } \\
6 \\
\end{array}$} & \multicolumn{2}{|c|}{$\begin{array}{c}\text { Regional Chain } \\
7 \\
\end{array}$} & \multicolumn{2}{|c|}{$\begin{array}{c}\text { Independent } \\
8 \\
\end{array}$} \\
\hline & $\begin{array}{l}\mathrm{R}^{2} \\
\mathrm{WPL}_{1}\end{array}$ & $\begin{array}{r}.04 \\
-.24\end{array}$ & $\begin{array}{l}\mathrm{R}^{2} \\
\mathrm{SP} \\
\mathrm{WPL}_{4}\end{array}$ & $\begin{array}{r}.66^{* *} \\
23.09^{* *} \\
.76^{* *}\end{array}$ & $\begin{array}{l}\mathrm{R}^{2} \\
\mathrm{SP} \\
\mathrm{WPL} \mathrm{L}_{6}\end{array}$ & $\begin{array}{c}.46^{* *} \\
26.27^{* *} \\
-.36^{*}\end{array}$ & $\begin{array}{l}\mathrm{R}^{2} \\
\mathrm{SP}\end{array}$ & $\begin{array}{r}.31^{* *} \\
25.03^{* *}\end{array}$ & $\begin{array}{l}\mathrm{R}^{2} \\
\mathrm{WPL}_{6}\end{array}$ & $\begin{array}{l}.23^{* *} \\
.48^{* *}\end{array}$ \\
\hline Ground chuck & $\begin{array}{l}\mathrm{R}^{2} \\
\mathrm{SP}\end{array}$ & $26.07^{* *}$ & $\begin{array}{l}\mathrm{R}^{2} \\
\mathrm{WP} \\
\mathrm{WPL} \mathrm{L}_{2}\end{array}$ & $\begin{array}{c}.36^{* *} \\
-1.64 \\
2.19^{* *}\end{array}$ & $\begin{array}{l}\mathrm{R}^{2} \\
\mathrm{WPL}_{4}\end{array}$ & $\begin{array}{r}.36^{* *} \\
-.40^{* *}\end{array}$ & $\begin{array}{l}\mathrm{R}^{2} \\
\mathrm{WPL} \mathrm{L}_{6}\end{array}$ & $\begin{array}{r}.24^{* *} \\
-.32^{* *}\end{array}$ & $\begin{array}{l}\mathrm{R}^{2} \\
\mathrm{SP}\end{array}$ & $\begin{array}{l}.17^{*} \\
5.51^{*}\end{array}$ \\
\hline Ground round & $\begin{array}{l}\mathrm{R}^{2} \\
\mathrm{WP} \\
\mathrm{WPL}_{3}\end{array}$ & $\begin{array}{c}.22^{*} \\
-.74^{*} \\
.43\end{array}$ & $\begin{array}{l}\mathrm{R}^{2} \\
\mathrm{WP} \\
\mathrm{WPL}_{2}\end{array}$ & $\begin{array}{c}.21 \\
-1.94^{*} \\
1.95^{*}\end{array}$ & $\begin{array}{l}\mathrm{R}^{2} \\
\mathrm{SP} \\
\mathrm{WPL}_{4}\end{array}$ & $\begin{array}{r}.75^{* *} \\
9.38^{* *} \\
-.29^{* *}\end{array}$ & $\begin{array}{l}\mathrm{R}^{2} \\
\mathrm{SP} \\
\mathrm{WPL}_{4} \\
\mathrm{WPL}_{5}\end{array}$ & $\begin{array}{r}.80^{* *} \\
12.14^{* *} \\
-.96^{* *} \\
.53\end{array}$ & & \\
\hline $\begin{array}{l}\text { Porterhouse } \\
\text { steak }\end{array}$ & $\begin{array}{l}\mathrm{R}^{2} \\
\text { WPLs }\end{array}$ & $\begin{array}{r}.61^{* *} \\
-3.08^{* *}\end{array}$ & $\begin{array}{l}\mathrm{R}^{2} \\
\mathrm{SP}\end{array}$ & $66.13^{* *}$ & $\begin{array}{l}\mathrm{R}^{2} \\
\mathrm{SP} \\
\mathrm{WPL} \mathrm{L}_{2}\end{array}$ & $\begin{array}{r}.95^{* *} \\
82.52^{* *} \\
-.63^{* *}\end{array}$ & $\begin{array}{l}\mathrm{R}^{2} \\
\mathrm{SP}\end{array}$ & $\begin{array}{r}.27^{* *} \\
72.22^{* *}\end{array}$ & $\begin{array}{l}\mathrm{R}^{2} \\
\mathrm{WPL} \mathrm{L}_{4}\end{array}$ & $\begin{array}{r}.14 \\
-1.05\end{array}$ \\
\hline Round steak & $\begin{array}{l}\mathrm{R}^{2} \\
\mathrm{SP}\end{array}$ & $\begin{array}{r}.92^{* *} \\
91.04^{* *}\end{array}$ & $\begin{array}{l}\mathrm{R}^{2} \\
\mathrm{WP} \\
\mathrm{SP}\end{array}$ & $\begin{array}{r}.98^{* *} \\
.72^{*} \\
104.13^{* *}\end{array}$ & $\begin{array}{l}\mathrm{R}^{2} \\
\mathrm{SP} \\
\mathrm{WPL} L_{2}\end{array}$ & $\begin{array}{l}.77^{* *} \\
64.44^{* *} \\
-.93\end{array}$ & $\begin{array}{l}\mathrm{R}^{2} \\
\mathrm{SP} \\
\mathrm{WPL}_{1}\end{array}$ & $\begin{array}{r}.89^{* *} \\
66.30^{* *} \\
-1.00^{* *}\end{array}$ & $\begin{array}{l}\mathrm{R}^{2} \\
\mathrm{SP}\end{array}$ & $\begin{array}{r}.43^{* *} \\
47.87^{* *}\end{array}$ \\
\hline
\end{tabular}


Table 12 Continued.

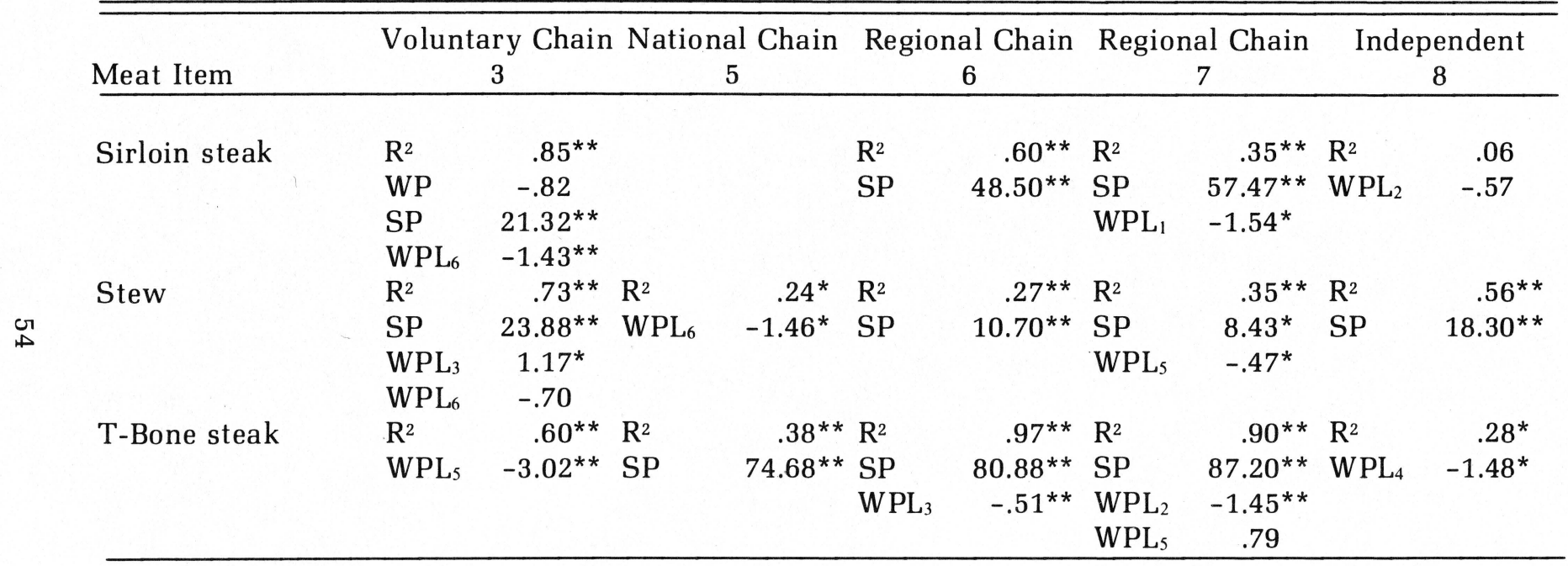

*Significant @ =.05

**Significant @=.01

${ }^{a}$ Blank space indicates that the variable was not included in this model. 
Blank Page in Original Bulletin 
